\title{
Approximation and estimation of very small probabilities of multivariate extreme events
}

\author{
Cees de Valk ${ }^{1}$
}

Received: 6 March 2015 / Revised: 18 March 2016 / Accepted: 7 April 2016 /

Published online: 25 April 2016

(C) The Author(s) 2016. This article is published with open access at Springerlink.com

\begin{abstract}
This article discusses modelling of the tail of a multivariate distribution function by means of a large deviation principle (LDP), and its application to the estimation of the probability $p_{n}$ of a multivariate extreme event from a sample of $n$ iid random vectors, with $p_{n} \in\left[n^{-\tau_{2}}, n^{-\tau_{1}}\right]$ for some $\tau_{1}>1$ and $\tau_{2}>\tau_{1}$. One way to view the classical tail limits is as limits of probability ratios. In contrast, the tail LDP provides asymptotic bounds or limits for log-probability ratios. After standardising the marginals to standard exponential, tail dependence is represented by a homogeneous rate function $I$. Furthermore, the tail LDP can be extended to represent both dependence and marginals, the latter implying marginal log-Generalised Weibull tail limits. A connection is established between the tail LDP and residual tail dependence (or hidden regular variation) and a recent extension of it. Under a smoothness assumption, they are implied by the tail LDP. Based on the tail LDP, a simple estimator for very small probabilities of extreme events is formulated. It avoids estimation of $I$ by making use of its homogeneity. Strong consistency in the sense of convergence of log-probability ratios is proven. Simulations and an application illustrate the difference between the classical approach and the LDP-based approach.
\end{abstract}

Keywords Multivariate extremes $\cdot$ Large deviation principle $\cdot$ Residual tail dependence $\cdot$ Hidden regular variation - Log-GW tail limit - Generalised Weibull tail limit

AMS 2000 Subject Classifications $60 \mathrm{~F} 10 \cdot 60 \mathrm{G} 70 \cdot 62 \mathrm{G} 32$

Cees de Valk

C.F.deValk@uvt.nl; ceesfdevalk@gmail.com

1 CentER, Tilburg University, P.O. Box 90153, 5000 LE Tilburg, The Netherlands 


\section{Introduction}

In this article, we will consider estimation of very small probabilities $p_{n}$ of multivariate extreme events from a sample of size $n$, with

$$
p_{n} \in\left[n^{-\tau_{2}}, n^{-\tau_{1}}\right] \quad \text { with } \quad \tau_{2}>\tau_{1}>1,
$$

motivated by applications requiring quantile estimates for $p_{n} \ll 1 / n$ in e.g. flood protection and more generally, natural hazard assessment, and in operational risk assessment for financial institutions. Multivariate events with such low probabilities are also relevant to these fields of application. Examples are breaching of a flood protection consisting of multiple sections differing in exposure, design and maintenance along a shoreline or river bank (Steenbergen et al. 2004), damage to an offshore structure caused by the combined effects of multiple environmental loads like water level, wave height, etc. (ISO 2005), and operational losses suffered by banks in different business lines and due to various types of events (Embrechts and Puccetti 2007).

Most work on estimation of probabilities of extreme events is based on the regularity assumption that the distribution function $F$ is in the domain of attraction of some extreme value distribution function (de Haan and Ferreira 2006; Resnick 1987). In the univariate case, this is equivalent to the generalised Pareto (GP) tail limit

$$
\lim _{t \rightarrow \infty} t(1-F(x w(t)+U(t)))=1 / h_{\gamma}^{-1}(x), \quad x \in h_{\gamma}((0, \infty))
$$

for some positive function $w$, with $U(t):=F^{-1}(1-1 / t)$ and for $\lambda>0$,

$$
h_{\gamma}(\lambda):= \begin{cases}\left(\lambda^{\gamma}-1\right) / \gamma & \text { if } \gamma \neq 0 \\ \log \lambda & \text { if } \gamma=0\end{cases}
$$

for some $\gamma \in \mathbb{R}$, the extreme value index. In the multivariate case, with $F$ the distribution function of a random vector $X=\left(X_{1}, . ., X_{m}\right)$ with continuous marginals $F_{1}, . ., F_{m}$, it implies that each marginal satisfies the GP tail limit (1.2) and that $V:=\left(V_{1}, . ., V_{m}\right)$, the random vector with standard Pareto marginals with

$$
V_{j}:=\left(1-F_{j}\left(X_{j}\right)\right)^{-1}
$$

for $i=1, . ., m$, satisfies

$$
\lim _{t \rightarrow \infty} t P(V \in t A)=v(A)
$$

for every Borel set $A \subset[0, \infty)^{m}$ such that $\inf _{x \in A} \max \left(x_{1}, . ., x_{m}\right)>0$ and $v(\partial A)=$ 0 , with $v$ a measure satisfying $v(A a)=a^{-1} v(A)$ for all these $A$ and all $a>0$. Based on the GP tail limit and the properties of the exponent measure $v$, estimators for probabilities have been formulated; e.g. Smith et al. (1990), Coles and Tawn (1991, 1994), Joe et al. (1992), Bruun and Tawn (1998), de Haan and Sinha (1999), Drees and de Haan (2015).

If the maxima of some components of $X$ under consideration are asymptotically independent, these estimators may produce invalid results. To alleviate this problem, residual tail dependence (RTD), also known as hidden regular variation, was introduced as an additional regularity assumption on the tail of the multivariate survival function $F^{c}$, defined by $F^{c}(x)=P\left(X_{i}>x_{i}, i=1, . ., m\right)$; e.g. Ledford and Tawn 
(1996, 1997, 1998), Peng (1999), Resnick (2002), Draisma et al. (2004) and Heffernan and Resnick (2005). This model was recently extended in Wadsworth and Tawn (2013). Another approach, based on conditional limits, was proposed in Heffernan and Tawn (2004) and Heffernan and Resnick (2007).

The first-order tail regularity conditions (1.2) and (1.5) can be seen as limiting relations for probability ratios. As such, they only allow estimation of probabilities $p_{n}$ vanishing slowly enough, that is,

$$
p_{n} \geq \lambda k_{n} / n
$$

for some $\lambda>0$ and some intermediate sequence ${ }^{1}\left(k_{n}\right)$; therefore, $n p_{n} \rightarrow \infty$ as $n \rightarrow \infty$. For an iid sample, the empirical probability $\hat{p}_{n}$ is an unbiased estimator for such $p_{n}$, satisfying that $\hat{p}_{n} / p_{n} \stackrel{p}{\rightarrow} 1$ (from the binomial distribution of $n \hat{p}_{n}$ ). Therefore, estimators for these $p_{n}$ which make use of tail regularity can at best achieve a reduction in variance when compared to $\hat{p}_{n}$. To allow tail extrapolation to be carried further to more rapidly vanishing $p_{n}$, additional assumptions beyond (1.2) and (1.5) are introduced. Initially, e.g. in Smith et al. (1990), Coles and Tawn (1991, 1994) and Joe et al. (1992), the tail is assumed to follow the limiting distribution exactly above some thresholds, so likelihood methods can be employed. Later, e.g. in de Haan and Sinha (1999), Peng (1999), Drees and de Haan (2015), Draisma et al. (2004) and de Haan and Ferreira (2006), convergence to the limiting distribution and its effect on bias in estimates is explicitly considered. For the marginals, additional assumptions on convergence to the limit (1.2) in these articles are identical to or stronger than those invoked for univariate quantile estimation. ${ }^{2}$ However, the latter appear to be restrictive when $\gamma=0$, regardless of the precise nature of the assumption; see de Valk (2016), Proposition 1. For example, they exclude the normal and the lognormal distribution, but also for all $\alpha \in(0, \infty) \backslash\{1\}$ the distribution functions of $Y^{\alpha}$ with $Y$ exponentially distributed, and of $\exp \left((\log V)^{\alpha}\right)$ with $V$ Pareto distributed.

To overcome these limitations, we will consider a different approach in this paper. Rather than imposing additional assumptions on convergence beyond the first-order limits (1.2) and (1.5), we will attempt to replace them by different types of firstorder limits more suitable for the probability range (1.1). Let $\left(k_{n}\right)$ be an intermediate sequence satisfying $k_{n} \leq n^{c}$ for some $c \in(0,1)$. Then $\left(p_{n}\right)$ satisfying (1.1) does not satisfy (1.6), but

$$
\tau_{1} \leq \frac{\log p_{n}}{\log \left(k_{n} / n\right)} \leq \tau_{2} /(1-c)<\infty .
$$

This suggests that replacing the classical limits of probability ratios by limits of log-probability ratios could provide a framework for constructing estimators for probabilities of extreme events in the range (1.1).

In the next section, we address the limiting behaviour of log-probability ratios in the univariate case as introduction to the multivariate case. We will find that this behaviour is described by a large deviation principle (LDP) (see e.g. Dembo and

\footnotetext{
${ }^{1}$ An intermediate sequence $\left(k_{n}\right)$ satisfies that $k_{n} \rightarrow \infty$ and $k_{n} / n \rightarrow 0$ as $n \rightarrow \infty$.

${ }^{2}$ Common assumptions are strong second-order extended regular variation as in e.g. Theorem 4.3.1(1) of de Haan and Ferreira (2006) or the Hall class (Hall 1982).
} 
Zeitouni (1998)). It is generalised to the multivariate setting in Section 3. In Section 4, we establish a connection between this LDP and residual tail dependence and related assumptions. Section 5 returns to the basic LDP and applies it to formulate a simple estimator for probabilities of extreme events in the range (1.1) and to prove its consistency. In Section 6, this estimator is compared to its classical analogues in simulations, and an application of the LDP-based estimator is presented as illustration. Section 7 closes with a discussion of the results and of outstanding issues. Readers primarily interested in tail dependence could scan Section 2 for the approach and background, read the first part of Section 3 until Eq. 3.12, and then continue with Sections 4-7. Lemmas can be found in Section 8.

The following notation is adopted: Id denotes the identity. The interior of a set $S$ is denoted by $S^{o}$ and its closure by $\bar{S}$. The image of a set $S$ under a function $f$ is written as $f(S)$. The infimum of an (extended) real function $f$ over $S$ is written as inf $f(S)$; by convention, $\inf \{\emptyset\}:=\infty$. To avoid tedious repetition, expressions of the form $a \leq \liminf _{y \rightarrow \infty} f(y) \leq \limsup _{y \rightarrow \infty} f(y) \leq b$ are abbreviated to $a \leq \liminf _{y \rightarrow \infty} f(y) \leq \limsup _{y \rightarrow \infty} \cdots \leq b$.

\section{Introducing the tail LDP: the univariate case}

We begin by examining the univariate case in order to become acquainted with a particular type of large deviation principle (LDP) as a model of the tail of a distribution function.

Let $X$ be a real-valued random variable and let $\left\{b_{y}, y>0\right\}$ be a family of real functions such that for $D \subset[0, \infty), b_{y}(D)$ becomes more extreme in some sense when $y$ is increased. In line with the classical limits (e.g. (1.2)), we could consider an affine function for $b_{y}$, i.e., $b_{y}(x)=r(y)+g(y) x$, with $r$ some nondecreasing function and $g$ some measurable positive function. Instead, for a reason to be explained later, we assume that $F(0)<1$ and consider

$$
b_{y}(x)=r(y) \mathrm{e}^{g(y) x}
$$

with $g$ and $r$ as above and $r(\infty)>0$. We examine the limiting behaviour of

$$
\frac{1}{y} \log P\left(X \in b_{y}(D)\right)
$$

as $y \rightarrow \infty$. Substituting $y_{n}=-\log \left(k_{n} / n\right)$ for $y$, this determines the behaviour of the $\log$-probability ratio in Eq. 1.7 with $p_{n}=P\left(X \in b_{y_{n}}(D)\right)$ as $n \rightarrow \infty$.

Generally speaking, normalised logarithms of probabilities like (2.2) do not need to satisfy limits, so we only assume that ${ }^{3}$

$$
J\left(D^{o}\right) \leq \liminf _{y \rightarrow \infty} \frac{1}{y} \log P\left(X \in b_{y}(D)\right) \leq \limsup _{y \rightarrow \infty} \ldots \leq J(\bar{D})
$$

\footnotetext{
${ }^{3}$ See the end of Section 1 for the notation employed here.
} 
for (at least) $D=(x, \infty)$ for all $x \geq 0$, with $J$ some monotonic set function taking values in $[0, \infty]$. Noting that $\varphi(x):=-J((x, \infty))$ is nondecreasing in $x$, we have at every continuity point $x$ of $\varphi$ in $(0, \infty)$,

$$
\lim _{y \rightarrow \infty} \frac{1}{y} \log \left(1-F\left(\mathrm{e}^{g(y) x} r(y)\right)\right)=-\varphi(x) .
$$

Let $q$ be the left-continuous inverse of $-\log (1-F)$, so

$$
q:=F^{-1}\left(1-\mathrm{e}^{-\mathrm{Id}}\right)=U \circ \exp .
$$

Assume that $\varphi$ is not constant. By Lemma 1.1.1 of de Haan and Ferreira (2006), Eq. 2.4 implies $\lim _{y \rightarrow \infty}(\log q(y \lambda)-\log r(y)) / g(y)=\varphi^{-1}(\lambda)$ at every continuity point of the left-continuous inverse $\varphi^{-1}$ of $\varphi$ in $(\varphi(0), \varphi(\infty))$. Therefore (cf. the proof of Theorem 1.1.3 in de Haan and Ferreira (2006)), we may take $r=q$ and choose $g$ measurable and such that $\varphi^{-1}(\lambda)=h_{\theta}(\lambda)$ for some real $\theta$ (see (1.3)). As a result,

$$
\lim _{y \rightarrow \infty} \frac{\log q(y \lambda)-\log q(y)}{g(y)}=h_{\theta}(\lambda), \quad \lambda>0,
$$

and from (2.4),

$$
\lim _{y \rightarrow \infty} \frac{1}{y} \log \left(1-F\left(\mathrm{e}^{g(y) x} q(y)\right)\right)=-h_{\theta}^{-1}(x), \quad x \in h_{\theta}((0, \infty)) .
$$

Equations 2.6 states that $\log q$ is extended regularly varying with index $\theta$. By Eq. 2.6, $\lim _{y \rightarrow \infty} g(y \lambda) / g(y)=\lambda^{\theta}$ for all $\lambda>0$, so $g \in R V_{\theta}$ ( $g$ is regularly varying with index $\theta$ ); see Appendix B of de Haan and Ferreira (2006). Now Eq. 2.3 can be fully specified:

Proposition 1 (a) Suppose that asymptotic bounds Eq. 2.3 apply to all D of the form $D=(x, \infty)$ with $x \geq 0$, with $J$ monotonic and $x \mapsto J((x, \infty))$ nonconstant. Then $g$ in Eq. 2.1 can be chosen such that Eq. 2.3 holds with $r=q$ and $J=-\inf h_{\theta}^{-1}(I d)$ for some $\theta \in \mathbb{R}$ for every Borel set $D \subset[0, \infty)$, i.e., for every Borel set $D \subset[0, \infty)$,

$$
\begin{aligned}
-\inf h_{\theta}^{-1}\left(D^{o}\right) & \leq \liminf _{y \rightarrow \infty} \frac{1}{y} \log P\left(\frac{\log X-\log q(y)}{g(y)} \in D\right) \\
& \leq \limsup _{y \rightarrow \infty} \ldots \leq-\inf h_{\theta}^{-1}(\bar{D}) ;
\end{aligned}
$$

(b) Equation 2.8 is equivalent to (2.6), which is equivalent to (2.7).

Proof We have proven that Eq. 2.3 for $D=(x, \infty)$ implies the equivalent limit relations (2.6) and (2.7), so it remains to be shown that (2.7) implies (2.8) for every Borel set $D \subset[0, \infty)$. The lower bound holds if $D^{o}$ is empty. Else, with $\alpha:=\inf h_{\theta}^{-1}\left(D^{o}\right) \geq 0$ and $\delta>0$ such that $\left(h_{\theta}(\alpha), h_{\theta}(\alpha+\delta)\right] \subset D^{o}$ and for every $\varepsilon \in(0, \delta / 2), P\left((\log X-\log q(y)) / g(y) \in D^{o}\right) \geq F\left(\mathrm{e}^{g(y) h_{\theta}(\alpha+\delta)} q(y)\right)-$ $F\left(\mathrm{e}^{g(y) h_{\theta}(\alpha)} q(y)\right) \geq \mathrm{e}^{-y(\alpha+\varepsilon)}-\mathrm{e}^{-y(\alpha+\delta-\varepsilon)} \geq \mathrm{e}^{-y(\alpha+\varepsilon)}\left(0 \vee 1-\mathrm{e}^{-y(\delta-2 \varepsilon)}\right)$, provided that $y$ is large enough, as a consequence of (2.7). As $\delta>0$ is arbitrary, this implies 
the lower bound in (2.8). The proof of the upper bound is similar and is therefore omitted.

The pair of equivalent limit relations (2.6) and (2.7) was named the logGeneralised Weibull (log-GW) tail limit in de Valk (2016), where it was proposed as a model for estimating high quantiles for probabilities in the range (1.1), as an alternative to the more familiar GP tail limit. If $\theta=0$ and $g(y) \rightarrow g_{\infty}>0$ as $y \rightarrow \infty$, it reduces to the Weibull tail limit; see e.g. Broniatowski (1993) and Klüppelberg (1991).

The log-GW tail limit looks deceptively similar to a GP tail limit, but it is a very different object, primarily due to the logarithm in Eq. 2.7 (or equivalently, due to the exponent in (2.5)). Its domain of attraction covers a wide range of tail weights: a class of light tails having finite endpoints, tails with Weibull limits (such as the normal distribution), all tails with classical Pareto tail limits and, more generally, with logWeibull tail limits. For the latter, $F$ o exp satisfies a Weibull tail limit; an example is the lognormal distribution. For estimation of high quantiles with probabilities (1.1) of distribution functions within the domain of attraction of the GP limit with $\gamma=0$, the log-GW tail limit offers a continuum of limits instead of just one; as a consequence, it is much more widely applicable (see de Valk (2016)). Readers more comfortable with classical tail limits may consider focusing on tails with a Pareto tail limit $(\gamma>0)$, which have a log-GW limit with $\theta=1\left(\operatorname{so} h_{\theta}(\lambda)=\lambda-1\right)$ and $g(y)=\gamma y$. This may make reading of the rest of the article easier.

An expression of the form Eq. 2.8 is an example of a large deviation principle 4 (LDP); see Section 1.2 of Dembo and Zeitouni (1998) for a general background. The rate function of the LDP (2.8) is $h_{\theta}^{-1}$. The bounds provided by an LDP are crude; for example, they are unaffected by multiplying the probability in Eq. 2.8 by a positive number. One could see this as the price to be paid for approximating probabilities over a very wide range. More precise bounds may exist, but such cases should be regarded as the exception rather than the rule. Observe also that the bounds do not involve integration and in fact, most of $D$ does not even matter to the values of the bounds. The LDP (2.8) reduces to a limit only if $D$ satisfies $\inf h_{\theta}^{-1}\left(D^{o}\right)=$ $\inf h_{\theta}^{-1}(\bar{D})$; such a $D$ is called a continuity set of the rate function.

Had we considered events of the form $b_{y}(x)=r(y)+g(y) x$ instead of Eq. 2.1, then in the same way as above, we would have arrived at a different tail limit, the GW limit defined by replacing $\log q$ by $q$ in Eq. 2.6 (see de Valk (2016)). Its domain of attraction covers a much more limited range of tail weights. Furthermore, if $F(0)<$ 1, then the GW limit implies a log-GW limit (cf. the proof of Lemma 3.5.1 in de Haan and Ferreira (2006)). Therefore, to ensure that the results of this article are sufficiently widely applicable, we focus on the log-GW limit.

\footnotetext{
${ }^{4}$ An LDP on a topological space $\mathcal{T}$ is an expression of the form Eq. 2.8 with

$P((\log X-\log q(y)) / g(y) \in D)$ generalised to $\mu_{y}(D)$, with $\left\{\mu_{y}, y>0\right\}$ some family of probability measures on the Borel $\sigma$-algebra, and $h_{\theta}^{-1}$ generalised to some rate function (= lower semicontinuous function) $I$; the expression is supposed to hold for every Borel set $D$ in $\mathcal{T}$.
} 
The events considered in Eq. 2.8 with $D \subset[0, \infty)$ imply that $X$ is in the interval ${ }^{5}$ $[q(y), \infty)$ for $q(y)>0$. In a multivariate setting, it would be desirable to extend this interval to $\mathbb{R}$, since a multivariate event could be extreme in one variable, but not in some other variable. This can be accomplished using a trick: define an approximation $\tilde{q}_{y}$ of $q(\operatorname{see}(2.5))$ for $y \in q^{-1}((0, \infty))$ by

$$
\tilde{q}_{y}(z):= \begin{cases}q(z) & \text { if } z \leq y \\ q(y) \mathrm{e}^{g(y) h_{\theta}(z / y)} & \text { if } z>y,\end{cases}
$$

so for $z>y, \tilde{q}_{y}(z)$ is the log-GW tail approximation; for $z \leq y$, it is exact.

A random variable $Y$ with the standard exponential distribution satisfies

$$
-\inf A^{o} \leq \liminf _{y \rightarrow \infty} \frac{1}{y} \log P(Y \in A y) \leq \limsup _{y \rightarrow \infty} \ldots \leq-\inf \bar{A}
$$

for every Borel set $A \subset[0, \infty)$, which can be proven in a similar manner as Proposition 1 . If $F$ is continuous, then $Y=-\log (1-F(X))$ has the standard exponential distribution and $q$ is increasing, so we can substitute $P(X \in q(A y))$ for $P(Y \in A y)$ in (2.10). Under the assumptions of Proposition 1, we can substitute $P\left(X \in \tilde{q}_{y}(A y)\right)$ for $P(Y \in A y)$ in Eq. 2.10 as well, extending (2.8) to

$$
-\inf A^{o} \leq \liminf _{y \rightarrow \infty} \frac{1}{y} \log P\left(X \in \tilde{q}_{y}(A y)\right) \leq \limsup _{y \rightarrow \infty} \ldots \leq-\inf \bar{A}
$$

for every Borel set $A \subset[0, \infty)$ :

Proposition 2 If $F$ is continuous, then (2.8), (2.6) and (2.7) are all equivalent to (2.11).

Proof Equivalence of Eqs. 2.6, 2.7 and 2.8 follows from Proposition 1(b). If $A^{o} \cap$ $(-\infty, 1)$ is nonempty, then $P\left(X \in \tilde{q}_{y}(A y)\right) \geq P\left(Y \in\left(A^{o} \cap(-\infty, 1)\right) y\right)$ and the lower bound in Eq. 2.11 follows from (2.10). If not, then by Eq. 2.9, $P(X \in$ $\left.\tilde{q}_{y}(A y)\right) \geq P\left(X \in \tilde{q}_{y}\left(A^{o} y\right)\right)=P\left((\log X-\log q(y)) / g(y) \in h_{\theta}\left(A^{o}\right)\right)$ with $h_{\theta}\left(A^{o}\right) \subset[0, \infty)$, so Proposition 1 implies the lower bound in (2.11). The upper bound is proven similarly. To show that Eq. 2.11 implies (2.7) for $x \in h_{\theta}((1, \infty))$, take $A=[\lambda, \infty)$ for $\lambda \geq 1$; it can be extended to $x \in h_{\theta}((0, \infty))$ by a standard argument.

When restricting $A$ to $[1, \infty)$, Eq. 2.11 is equivalent to Eq. 2.8 for $D=h_{\theta}^{-1}(A)$. With $A$ in $[0, \infty)$, therefore, Eq. 2.11 provides the intended generalisation of (2.8). Note that the $\log$-GW index $\theta$ and auxiliary function $g$ are now hidden in the approximation $\tilde{q}_{y}$ in (2.9). However, they are as essential in Eq. 2.11 as they are in the more explicit (2.8).

\footnotetext{
${ }^{5}$ Depending on $\theta$, we can extend this somewhat to $\left[q(y) \mathrm{e}^{-c g(y)}, \infty\right)$ for some $c>0$; see (2.7).
} 


\section{Bounds and limits for probabilities of multivariate tail events}

For the univariate tail, we obtained the LDP (2.11) in a form which closely resembles (2.10) for the standard exponential distribution. This suggests that for a multivariate generalisation, we examine first the case of a random vector $Y:=\left(Y_{1}, . ., Y_{m}\right)$ with distribution function having standard exponential marginals. A straightforward multivariate generalisation of the LDP (2.10) would be

$$
-\inf I\left(A^{o}\right) \leq \liminf _{y \rightarrow \infty} \frac{1}{y} \log P(Y / y \in A) \leq \limsup _{y \rightarrow \infty} \ldots \leq-\inf I(\bar{A})
$$

for every Borel set $A \subset[0, \infty)^{m}$, with $I$ some rate function; we may regard (3.1) as the analogue of the classical expression (1.5). Further on, we will prove that Eq. 3.1 holds if

$I(x):=-\inf _{\varepsilon>0} \liminf _{y \rightarrow \infty} \frac{1}{y} \log P\left(Y / y \in B_{\varepsilon}(x)\right)=-\inf _{\varepsilon>0} \limsup _{y \rightarrow \infty} \frac{1}{y} \log P\left(Y / y \in B_{\varepsilon}(x)\right)$,

with $B_{\varepsilon}(x):=\left\{x^{\prime} \in \mathbb{R}^{m}:\left\|x-x^{\prime}\right\|_{\infty}<\epsilon\right\}$ the open ball of radius $\varepsilon>0$ with centre $x \in \mathbb{R}^{m}$. For now, we turn to the rate function $I$, defined by Eq. 3.2 as some kind of limiting density, with the probability of an open ball replaced by its logarithm. Several properties of $I$ follow immediately from Eq. 3.2 and the exponential marginals of $Y$. For every $\varepsilon>0$ and $x \in \mathbb{R}^{m}$ with $x_{j}=\lambda>0$ for some $j \in\{1, \ldots, m\}$, $\frac{1}{y} \log P\left(Y / y \in B_{\varepsilon}(x)\right) \leq \frac{1}{y} \log P\left(Y_{j} / y>(\lambda-\varepsilon)\right)=\varepsilon-\lambda$, so

$$
I(x) \geq \max _{j \in\{1, . ., m\}} x_{j}, \quad x \in \mathbb{R}^{m} .
$$

This implies that $I$ is a good rate function, meaning that $I^{-1}([0, a])$ is compact for every $a \in[0, \infty)$. Also, since $B_{\varepsilon}(x \lambda)=\lambda B_{\varepsilon / \lambda}(x)$,

$$
I(x \lambda)=\lambda I(x), \quad \lambda>0, x \in \mathbb{R}^{m} .
$$

Furthermore, $I(0)=0$, since $P\left(\|Y\|_{\infty} \leq y \varepsilon\right) \geq 1-m P\left(Y_{1}>\varepsilon y\right)=1-m e^{-\varepsilon y}$ in (3.2), and $I(x)=\infty$ whenever $\min \left(x_{1}, . ., x_{m}\right)<0$.

Remark 1 By Eq. 3.4, $I(x)=\varrho(x) I(x / \varrho(x))$ for every $x \in \mathbb{R}^{m} \backslash\{0\}$ and every norm $\varrho$ on $\mathbb{R}^{m}$. This gives for every norm a "spectral representation" of $I$, analogous to the spectral measures in classical extreme value theory (e.g. de Haan and Ferreira (2006), Section 6.1.4). For example, in the bivariate case, the rate function can be represented on $[0, \infty)^{2} \backslash\{0\}$ by $I(x)=\left(x_{1}+x_{2}\right) \psi\left(x_{2} /\left(x_{1}+x_{2}\right)\right)$ with $\psi(t):=I(1-t, t)$ for $t \in[0,1]$, so by Eq. 3.3, it satisfies $\psi(t) \geq \max (t, 1-t)$ for all $t \in[0,1]$. The similarity of $\psi$ to the dependence function $A$ of Pickands (1981) may be misleading, as a rate function defined by Eq. 3.2 and a distribution function are very different objects. Besides satisfying $A(t) \geq \max (t, 1-t)$ for all $t \in[0,1]$, Pickands' function $A$ is convex, and $A(1)=A(0)=1$. These latter conditions do not need to apply to $\psi$. 
Example 1 Let $X \sim \mathcal{N}(0, V)$ with $V$ an $m \times m$ positive-definite matrix with unit diagonal; let $W:=V^{-1}$. Then

$$
I(x)=\sum_{1 \leq i, j \leq m} w_{j i} \sqrt{x_{i} x_{j}}, \quad x \in[0, \infty)^{m} .
$$

In the bivariate case with $v_{12}=v_{21}=: \rho, I(x)=\left(x_{1}+x_{2}-2 \rho \sqrt{x_{1} x_{2}}\right) /\left(1-\rho^{2}\right)$, so $\psi(t)=(1-2 \rho \sqrt{t(1-t)}) /\left(1-\rho^{2}\right)$. If $\rho>0$, then $I$ is convex and therefore, $\psi$ is convex. Figure 1 shows contour plots of $I$ for $\rho=0.8$ (left) and $\rho=0.2$ (middle). On the right, the function $\psi$ is plotted for these two values of $\rho$; for both, $\psi(1)=\psi(0)>1$.

Remark 2 If $\lim _{y \rightarrow \infty} P\left(\min _{j=1, . ., m} Y_{j}>y\right) / P\left(Y_{1}>y\right)>0$, then $I(1)=1$ with $1=(1, . ., 1)$; the converse is not true. In the bivariate case, $I(1)=1$ implies that $\psi\left(\frac{1}{2}\right)=\frac{1}{2}$ for $\psi$ in Remark 1. It does not fix $\psi(t)$ at other $t \in[0,1]$. For example, for the bivariate normal distribution with standard marginals (see Example 1) and with $\rho=1, \psi(t)=\infty$ for all $t \in[0,1] \backslash\left\{\frac{1}{2}\right\}$, but for a positive mixture of this distribution function with a similar one with $\rho=r<1, \psi(t)=(1-2 r \sqrt{t(1-t)}) /\left(1-r^{2}\right)$ for all $t \in[0,1] \backslash\left\{\frac{1}{2}\right\}$.

Remark 3 If $I$ is subadditive, then by Eq. 3.4, it is convex, and furthermore, by Eq. 3.3 , it is a norm. Again, this condition does not need to be satisfied in general.

$$
\begin{aligned}
& \text { Since } P\left(Y_{1}>y \alpha\right) \leq P\left(\|Y\|_{\infty}>y \alpha\right) \leq m P\left(Y_{1}>y \alpha\right), \\
& \lim _{y \rightarrow \infty} \frac{1}{y} \log P\left(\|Y\|_{\infty} / y>\alpha\right)=-\alpha, \quad \alpha \geq 0,
\end{aligned}
$$

so the tail of the maximum of $Y_{1}, . ., Y_{m}$ satisfies the same limit relation as the tails of each of $Y_{1}, . ., Y_{m}$ individually. This implies that the family of probability measures
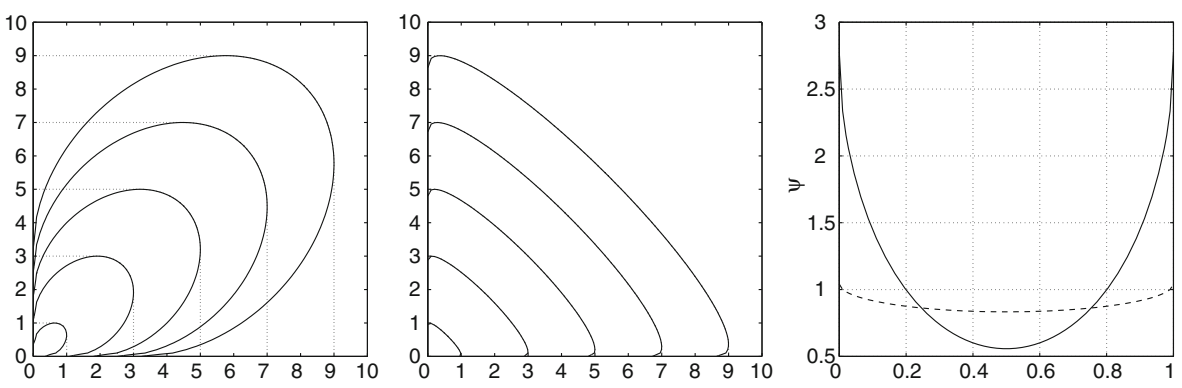

Fig. 1 Left and middle contours of the rate function $I$ (drawn) and function $\kappa$ (dotted; see Section 4) for the bivariate normal distribution with standard marginals and $\rho=0.8$ (left) and $\rho=0.2$ (middle). Right function $\psi$ (see text) for $\rho=0.8$ (drawn) and $\rho=0.2$ (dashed) 
corresponding to the random variables $\{Y / y, y>0\}$ is exponentially tight (Dembo and Zeitouni 1998): for every $\alpha<\infty$, a compact $E_{\alpha} \subset \mathbb{R}^{m}$ exists such that

$$
\limsup _{y \rightarrow \infty} \frac{1}{y} \log P\left(Y / y \in E_{\alpha}^{c}\right)<-\alpha,
$$

which follows from Eq. 3.5 when taking $E_{\alpha}=\left\{x \in \mathbb{R}^{m}:\|x\|_{\infty} \leq \alpha+\varepsilon\right\}$ for some $\varepsilon>0$. As a consequence,

Proposition 3 Let $Y:=\left(Y_{1}, \ldots, Y_{m}\right)$ be a random vector with standard exponential marginals. If it satisfies (3.2), then it satisfies (3.1) for all Borel $A \subset[0, \infty)^{m}$ with good rate function I satisfying (3.4), I(0) $=0$ and the marginal condition

$$
\inf _{x \in \mathbb{R}^{m}: x_{j}>\lambda} I(x)=\lambda, \quad \lambda \geq 0, j=1, \ldots, m .
$$

Proof By Theorem 4.1.11 in Dembo and Zeitouni (1998), Eq. 3.2 implies the weak LDP, i.e., the lower bound in Eq. 3.1 holds for all Borel $A$, and the upper bound of Eq. 3.1 holds for all compact $A$. Because of exponential tightness (3.6), this implies the LDP (3.1); see Lemma 1.2.18 in Dembo and Zeitouni (1998). Then Eq. 3.7 follows from Eq. 3.1 and the exponential marginals of $Y$.

Remark 4 Equation 3.3 is implied by (3.7).

For a continuity set $A$ of $I$ satisfying that inf $I(\bar{A})=\inf I\left(A^{o}\right)$, the bounds in (3.1) reduce to a limit:

$$
\lim _{y \rightarrow \infty} \frac{1}{y} \log P(Y \in A y)=-\inf I(A) .
$$

A sufficient condition for a set $A$ to be a continuity set of $I$ is that $I$ is continuous and $A \subset \overline{A^{o}}$. Homogeneity (3.4) of $I$ allows us to relax this condition: without assuming continuity of $I, A$ is a continuity set if inf $I(\bar{A})=I(x)$ for some $x \in \overline{A^{\circ}} \cap$ $\cup_{\lambda>0}\left(\lambda A^{o}\right)\left(\cup_{\lambda>0}\left(\lambda A^{o}\right)\right.$ is the smallest cone containing $\left.A^{o}\right)$. A bivariate example is sketched in Fig. 2. Let $A^{o}$ be the grey set; if $I$ attains its infimum over $\bar{A}$ on the part of its boundary drawn as a fat line (excluding the points indicated by circles), then $A$ is a continuity set. In the remainder of this article, we will discuss continuity sets of rate functions without considering the particular conditions which make them so.

It is straightforward to extend Proposition 3 to a random vector $X$ with a distribution function $F$ having continuous marginals $F_{1}, . ., F_{m}$. As in Eq. 2.5, let for $i=1, . ., m$,

$$
q_{i}:=F_{i}^{-1}\left(1-e^{-\mathrm{Id}}\right)
$$

and for every $x \in[0, \infty)^{m}$,

$$
Q(x):=\left(q_{1}\left(x_{1}\right), . ., q_{m}\left(x_{m}\right)\right) .
$$

Let $Y:=\left(Y_{1}, . ., Y_{m}\right)$ with for $j=1, . ., m$,

$$
Y_{j}:=-\log \left(1-F_{j}\left(X_{j}\right)\right),
$$




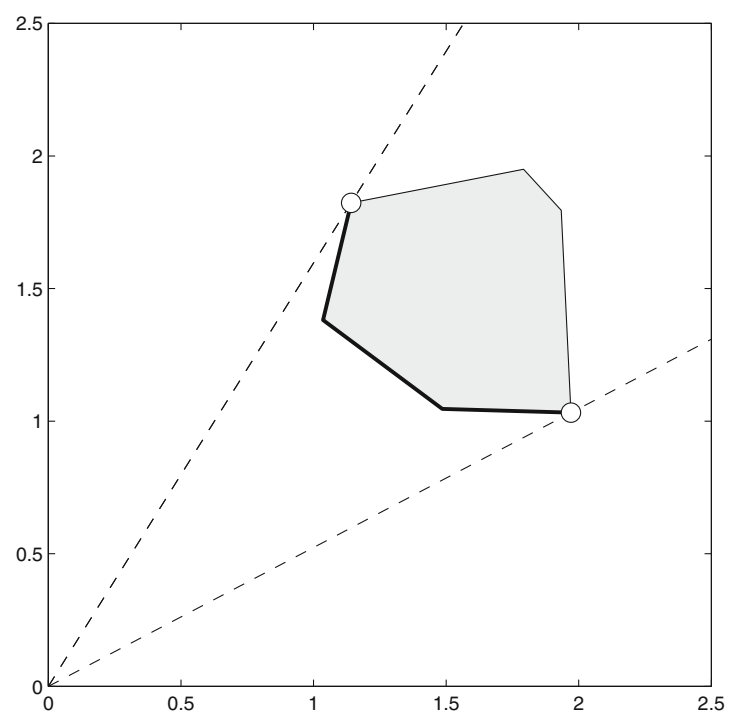

Fig. 2 Illustration of a continuity set of $I$ (see main text)

so $Y=Q^{-1}(X)$. Because $F_{1}, . ., F_{m}$ are continuous, $Y$ has exponential marginals. Almost surely, $X=Q(Y)$ with $Q$ defined by Eqs. 3.10 and 3.9. Since $Q$ is injective, $P(X \in Q(y A))=P(Y \in y A)$, so (3.1) is equivalent to

$$
-\inf I\left(A^{o}\right) \leq \liminf _{y \rightarrow \infty} \frac{1}{y} \log P(X \in Q(y A)) \leq \limsup _{y \rightarrow \infty} \ldots \leq-\inf I(\bar{A}) .
$$

Having obtained a multivariate version of Eq. 2.10, we are now ready to generalise the univariate tail LDP (2.8) and its extension (2.11) to the multivariate context. Concerning the latter, one would expect its multivariate generalisation to be like (3.12), with $Q$ replaced by an approximation. Let $F_{1}, . ., F_{m}$ satisfy log-GW tail limits with scaling functions $g_{1}, \ldots, g_{m}$ and $\log -\mathrm{GW}$ indices $\theta_{1}, . ., \theta_{m}$, respectively. As in Eq. 2.9, define marginal quantile approximations

$$
\tilde{q}_{j, y}(z):= \begin{cases}q_{j}(z) & \text { if } z \leq y \\ q_{j}(y) e^{g_{j}(y) h_{\theta_{j}}(z / y)} & \text { if } z>y\end{cases}
$$

for $y \in \cap_{j=1}^{m} q_{j}^{-1}((0, \infty))$, and let for all $x=\left(x_{1}, . ., x_{m}\right) \in[0, \infty)^{m}$,

$$
\tilde{Q}_{y}(x):=\left(\tilde{q}_{1, y}\left(x_{1}\right), \ldots, \tilde{q}_{m, y}\left(x_{m}\right)\right) .
$$

Theorem 1 Let the random vector $X=\left(X_{1}, \ldots, X_{m}\right)$ have distribution function $F$ with continuous marginals $F_{1}, . ., F_{m}$ having positive endpoints. 
(a) If $Y$ defined by Eq. 3.11 satisfies (3.2) and the marginals satisfy log-GW tail limits (2.6) with $q=q_{j}, g=g_{j}$ and $\theta=\theta_{j}$ for $j=1, . ., m$, then $X$ satisfies

$$
-\inf I\left(A^{o}\right) \leq \liminf _{y \rightarrow \infty} \frac{1}{y} \log P\left(X \in \tilde{Q}_{y}(y A)\right) \leq \limsup _{y \rightarrow \infty} \ldots \leq-\inf I(\bar{A})
$$

for every Borel set $A \subset[0, \infty)^{m}$, with $\tilde{Q}_{y}$ given by Eqs. 3.13 and 3.14 , and I a good rate function satisfying (3.4), (3.7) and $I(0)=0$.

(b) If $X$ satisfies (3.15) for every Borel set $A \subset[0, \infty)^{m}$ with $\tilde{Q}_{y}$ given by Eqs. 3.13 and 3.14 and with rate function I satisfying (3.7), then the marginals satisfy log-GW tail limits, and $Y$ defined by Eq. 3.11 satisfies (3.1) with good rate function I satisfying (3.4), (3.7) and $I(0)=0$.

The proof can be found in Section 8.1.

Remark 5 This theorem justifies viewing (3.1) as representation of tail dependence within the context of the LDP (3.15), which also represents the marginal tails. The relationship between the LDPs (3.15) and (3.1) is the large deviations analogue of a similar relationship in classical extreme value theory; compare e.g. Resnick (1987), Propositions 5.10 and 5.15.

From the multivariate generalisation of Eq. 2.11, we can now also derive a multivariate version of Eq. 2.8, equivalent to the restriction of Eq. 3.15 to $A \subset$ $[1, \infty)^{m}$ :

Corollary 1 Let $\theta:=\left(\theta_{1}, . ., \theta_{m}\right)$ and $H_{\theta}(z):=\left(h_{\theta_{1}}\left(z_{1}\right), . ., h_{\theta_{m}}\left(z_{m}\right)\right)$ for all $z \in$ $(0, \infty)^{m}$. Then Eq. 3.15 implies for every Borel set $D \in[0, \infty)^{m}$ :

$$
\begin{gathered}
-\inf I\left(H_{\theta}^{-1}\left(D^{o}\right)\right) \\
\leq \liminf _{y \rightarrow \infty} \frac{1}{y} \log P\left(\left(\frac{\log X_{1}-\log q_{1}(y)}{g_{1}(y)}, . ., \frac{\log X_{m}-\log q_{m}(y)}{g_{m}(y)}\right) \in D\right) \\
\leq \limsup _{y \rightarrow \infty} \ldots \leq-\inf I\left(H_{\theta}^{-1}(\bar{D})\right) .
\end{gathered}
$$

Proof See Section 8.1.

Note that Eq. 3.16 only addresses events within $\left(q_{1}(y), \infty\right) \times \ldots \times\left(q_{m}(y), \infty\right)$, which is "covered" by all marginal log-GW tail approximations simultaneously. Just as Eq. 2.8, it can be extended somewhat. However, the main interest of Eq. 3.16 is that it shows the multivariate tail LDP explicitly as a pair of asymptotic bounds for the probabilities of extreme events defined in terms of affinely normalised logarithms of the components of $X$. For applications in statistics, Eq. 3.15 should be more useful, as it applies also to events which are not simultaneously extreme in every component of $X$. 


\section{A connection to residual tail dependence and related models}

In this section, we digress from the main storyline to examine an interesting connection between the theory of Section 3 and earlier work on residual tail dependence (RTD) or hidden regular variation, introduced in Ledford and Tawn (1996, 1997, 1998) and studied in depth in Resnick (2002), amongst others. In the bivariate case, RTD offers a model of tail dependence within the classical domain of asymptotic independence of component-wise maxima (e.g. de Haan and Ferreira (2006), Section 7.6). For a random vector $X$ on $\mathbb{R}^{m}$ with continuous marginals $F_{1}, \ldots, F_{m}$, defining the random vector $V:=\left(V_{1}, \ldots, V_{m}\right)$ with standard Pareto-distributed variables by Eq. 1.4, one way to describe RTD is that for some positive function $S$ on $(0, \infty)^{m}$,

$$
\lim _{t \rightarrow \infty} \frac{P\left(V_{j}>t x_{j}, j=1, . ., m\right)}{P\left(V_{j}>t, j=1, . ., m\right)}=: S(x)>0, \quad x \in(0, \infty)^{m} .
$$

The limiting function $S$ satisfies $S(l)=1$, with 1 the vector in $\mathbb{R}^{m}$ with all its components equal to 1. Furthermore, the denominator in Eq. 4.1 must be regularly varying, so $S(\lambda l)=\lambda^{-1 / \eta}$ for all $\lambda>0$ with $\eta \in(0,1]$ the residual dependence index, and by Eq. 4.1,

$$
S(x \lambda)=\lambda^{-1 / \eta} S(x), \quad x \in(0, \infty)^{m}, \lambda>0 .
$$

Every regularly varying function $f \in R V_{\alpha}$ can be represented as

$$
f(y)=c(y) e^{\int_{y_{0}}^{y} a(t) t^{-1} d t}
$$

with $c(y) \rightarrow c_{0}>0$ and $a(y) \rightarrow \alpha$ as $y \rightarrow \infty$. A minor strengthening of regular variation is that $f$ satisfies the Von Mises condition (see e.g. Proposition 1.15 of Resnick (1987)), which means that $c$ in Eq. 4.3 can be taken equal to a positive number $c_{0}$; it implies that $f$ is differentiable with derivative $f^{\prime}(y)=a(y) f(y) / y$. Note that whenever the LDP (3.1) holds for $Y$ given by Eq. 3.11 and $\inf I(A) \in$ $(0, \infty)$ for a Borel continuity set $A$ of $I$, then the function $(y \mapsto-\log P(Y / y \in A))$ is in $R V_{1}$. Therefore, within the context of the LDP (3.1), the statement that $(y \mapsto$ $-\log P(Y / y \in A))$ satisfies the Von Mises condition makes sense as a smoothness condition. The following relates RTD to the tail LDP (3.1).

\section{Proposition 4 (a) RTD (4.1) implies}

$$
\lim _{y \rightarrow \infty} \frac{1}{y} \log P\left(Y / y \in(\lambda, \infty)^{m}\right)=-\lambda / \eta, \quad \lambda>0,
$$

with $\eta$ the residual dependence index of $X$.

(b) If $X$ satisfies the LDP (3.1) with the function $\left(y \mapsto-\log P\left(Y / y \in(1, \infty)^{m}\right)\right)$ satisfying the Von Mises condition, then Eq. 4.1 holds for $x=\lambda 1$ for all $\lambda>0$, with $S$ satisfying $S(1 \lambda)=\lambda^{-1 / \eta}$ and $\eta=1 / I(1)$.

Proof Define $Y_{\wedge}:=\min _{j \in\{1, \ldots, m\}} Y_{j}$, and let $H_{\wedge}$ be the distribution function of $Y_{\wedge}$. By Eq. 4.1, the survival function $1-H_{\wedge} \circ \log$ of the random variable $\exp Y_{\wedge}$ is 
regularly varying with index $-1 / \eta$. Therefore, $f:=1 /\left(1-H_{\wedge} \circ \log \right) \in R V_{\{1 / \eta\}}$, so by the Potter bounds (Bingham et al. 1987), for every $\varepsilon \in(0,1 / \eta)$, there is $z_{\varepsilon}>0$ such that $(1-\varepsilon)(x / z)^{1 / \eta-\varepsilon} \leq f(x) / f(z) \leq(1+\varepsilon)(x / z)^{1 / \eta+\varepsilon}$ for all $z \geq z_{\varepsilon}$ and $x \geq z$. Taking logarithms and substituting $\mathrm{e}^{y \lambda}$ for $x$ gives $\lim _{y \rightarrow \infty} y^{-1} \log f\left(\mathrm{e}^{y \lambda}\right) \rightarrow \lambda / \eta$ for all $\lambda>0$, so Eq. 4.4 follows. For (b), note that due to Eq. 3.4, the LDP (3.1) implies (4.4) with $\eta=1 / I(1)$, so $w(y):=-\log \left(1-H_{\wedge}(y)\right) \sim y / \eta$ as $y \rightarrow \infty$. Therefore, since $w$ satisfies the Von Mises condition, $w^{\prime}(y) \rightarrow 1 / \eta$ and by averaging, $w(y+r)-w(y) \rightarrow r / \eta$ as $y \rightarrow \infty$ for every $r \in \mathbb{R}$. This is equivalent to Eq. 4.1 for $x=\lambda l$ with $S(1 \lambda)=\lambda^{-1 / \eta}$ for every $\lambda>0$.

Proposition 4 shows that RTD implies a limited LDP-like condition and in turn, the LDP (3.1) with an additional smoothness condition implies an RTD-like condition.

Example 2 The bivariate normal $X$ of Example 1 satisfies the conditions for Proposition 4(b) with $I(1)=2 /(1+\rho)$. Indeed, Eq. 4.1 holds for $x=\lambda l$ for all $\lambda>0$, with $S(1 \lambda)=\lambda^{-1 / \eta}$ and $\eta=(1+\rho) / 2$; see Example Class 2(1) in Ledford and Tawn (1996).

If $\lim _{t \rightarrow \infty} t^{-1} P\left(V_{j}>t, j=1, . ., m\right)=0$, then there is a discrepancy between the "hidden" regularity of the survival function in $(0, \infty)^{m}$ described by Eq. 4.1 and the regularity of the marginals. In contrast, the LDP (3.1) provides a single consistent description of the multivariate tail which includes the marginal tails. Furthermore, the next theorem shows that under a smoothness assumption similar to the one in Proposition 4(b), the LDP (3.1) implies a useful extension of RTD. Let for all $a \in$ $\mathbb{R}^{m}$,

$$
A_{a}:=\left\{x \in \mathbb{R}^{m}: x_{j}>a_{j}, j=1, . ., m\right\} .
$$

Theorem 2 (a) Assume that the LDP (3.1) applies. To any Borel set $A \subset \mathbb{R}^{m}$ which is a continuity set of $I$ with $(y \mapsto-\log P(Y / y \in A))$ satisfying the Von Mises condition, the following limit relation applies:

$$
\lim _{t \rightarrow \infty} \frac{P(Y \in A \log (t \lambda))}{P(Y \in A \log t)}=\lambda^{-\inf I(A)}, \quad \lambda>0,
$$

with I satisfying (3.4), (3.7) and $I(0)=0$. In particular, for every $a \in[0, \infty)^{m}$ such that the function $\left(y \mapsto-\log P\left(Y_{j}>y a_{j}, j \in 1, . ., m\right)\right.$ satisfies the Von Mises condition,

$$
\lim _{t \rightarrow \infty} \frac{P\left(V_{j}>(t \lambda)^{a_{j}}, j=1, . ., m\right)}{P\left(V_{j}>t^{a_{j}}, j=1, . ., m\right)}=\lambda^{-\inf I\left(A_{a}\right)}, \quad \lambda>0 .
$$

(b) Equation 4.6 with $\inf I(A) \in(0, \infty)$ implies (3.8).

Proof For $A$ a continuity set of $I$, (3.1) implies (3.8), and (4.6) is obtained in the same manner as in the proof of Proposition 4(b). In particular, $A_{a}$ is a continuity set of $I$ for every $a \in[0, \infty)^{m}$. Therefore, substituting $A_{a}$ for $A$ in Eq. 4.6, we obtain (4.7). This proves (a). For (b), note that $f:=(t \mapsto 1 / P(Y \in A \log t)) \in R V_{\inf I(A)}$. Therefore, 
just as in the proof of Proposition 4(a), $\lim _{y \rightarrow \infty} y^{-1} \log f\left(\mathrm{e}^{y \lambda}\right) \rightarrow \lambda \inf I(A)$ for all $\lambda \geq 1$, which implies (3.8).

Combining (a) and (b) in Theorem 2, we see that under the Von Mises condition (for $A$ a Borel continuity set of $I$ ), the limit relation (4.6) for a probability ratio, and the limit relation (3.8) for the normalised logarithm of a probability are equivalent.

In the special case of $a=1$, Eq. 4.7 becomes equivalent to Eq. 4.1 with $x=\lambda 1$ and $\eta=1 / I(1)$, so on the diagonal, Eq. 4.7 and RTD (4.1) agree; elsewhere, they differ. Defining a function $\kappa$ by $\kappa(a):=\inf I\left(A_{a}\right)$ for every $a \in[0, \infty)^{m}$, Eq. 4.7 becomes identical to an extension of RTD recently introduced in Wadsworth and Tawn (2013). Wadsworth and Tawn (2013) proposed this assumption to close the possible gap between (4.1) and the regularity of the marginal tails. It is curious that this condition, requiring the existence of separate limits of the survival function along chosen paths, is derivable from the simple LDP (3.1).

The generalisation of Eq. 4.7 with $\inf I\left(A_{a}\right)$ replaced by $\kappa(a)$ to the apparently new limit relation (4.6) is not trivial. Another generalisation, proposed in Wadsworth and Tawn (2013), is

$$
\lim _{t \rightarrow \infty} \frac{P(Y \in B+a \log (t \lambda))}{P(Y \in B+a \log t)}=\lambda^{-\kappa(a)}, \quad \lambda>0,
$$

derived in Section 3.3 of Wadsworth and Tawn (2013) for the bivariate case under the assumption that $\kappa$ is differentiable and $a \in[0, \infty)^{m} \backslash\{0\}$ satisfies $\partial \kappa(a) / \partial a_{j}>0$ for $j=1, . ., m$. As noted in Wadsworth and Tawn (2013), $a$ in Eq. 4.8 would have to be chosen in an application. This would be no problem if the choice did not matter. However, the limiting behaviour of the probability of the event $Y \in B+a \log t$ as $t \rightarrow$ $\infty$ is determined by $a$ in Eq. 4.8 ; not by $B$. Therefore, for estimating probabilities of extreme events, Eq. 4.6 seems more promising than the local limits (4.8) for chosen $a$.

In Eq. 4.6, it is not $\kappa$, but the rate function $I$ which determines the attenuation rate. For any $a \in[0, \infty)^{m}, I(a)$ and $\kappa(a)$ are identical only if $I(a+x) \geq I(a)$ for all $x \in[0, \infty)^{m}$. This condition is rather restrictive, as a rate function resembles a density more than it resembles a survival function; see definition (3.2).

Example 3 As an illustration, let $X$ be bivariate normal with correlation coefficient $\rho$ as in Example 1 (Section 3). By Example 1(a,b) in Table 1 of Wadsworth and Tawn (2013), $\kappa(x)=I(x)$ if $\min \left(x_{1} / x_{2}, x_{2} / x_{2}\right)>\rho^{2}$ or if $\rho<0$ and $\min \left(x_{1}, x_{2}\right)>0$, and $\kappa(x)=\max \left(x_{1}, x_{2}\right)$ for all other $x \in[0, \infty)^{2}$. The left and middle panels of Fig. 1 display contours of $\kappa$ overlaying the contours of $I$ for $\rho=0.8$ and $\rho=0.2$. For $\rho=0.2$, contours of $\kappa$ largely overlap with those of $I$; for $\rho=0.8$, there are wide zones where the contours of $\kappa$ and $I$ differ.

\section{A simple estimator for very small probabilities}

We are now going to apply the theory of Section 3 to the problem of estimation of probabilities of extreme events $p_{n}$ satisfying (1.1) from $X^{(1)}, \ldots, X^{(n)}$, 
with $X^{(1)}, X^{(2)}, \ldots$ a sequence of iid copies of a random vector $X$ in $\mathbb{R}^{m}$ with distribution function $F$ having continuous marginals $F_{1}, \ldots, F_{m}$. Denoting the underlying probability space as $(\Omega, \mathcal{F}, \mathcal{P})$, let $\mathcal{F}_{n} \subset \mathcal{F}$ be the $\sigma$-algebra generated by $X^{(1)}, \ldots, X^{(n)}$.

Generalising (1.1) to $\tau_{2}>\tau_{1}>0$, consider events of the form $B_{n}:=Q(A \log n)$ with $A \subset[0, \infty)^{m}$ and $Q$ given by Eq. 3.10. Suppose that the tail LDP (3.1) applies. Then for every Borel set $A \subset[0, \infty)^{m}$ which is a continuity set of $I$ satisfying that $\inf I(A) \in(0, \infty)$, we have: $-\log P\left(X \in B_{n}\right)=-\log P(Y \in$ $A \log n) \sim(\log n) \inf I(A)$ and $-\log P\left(Q(Y / \ell) \in B_{n}\right)=-\log P(Y \in A \ell \log n) \sim$ $\ell(\log n) \inf I(A)$ for all $\ell>0$, so

$$
\log P\left(X \in B_{n}\right) \sim \ell^{-1} \log P\left(Q(Y / \ell) \in B_{n}\right), \quad \ell>0 .
$$

This suggests estimating the left-hand side of Eq. 5.1 by replacing $Q$ on the righthand side by an estimator $\hat{Q}_{n}$ and $Y$ by an estimator $\hat{Y}_{n}$, and then choosing $\ell$ small enough that $P\left(\hat{Q}_{n}\left(\hat{Y}_{n} / \ell\right) \in B_{n}\right)$ can be estimated nonparametrically by counting.

Estimation of $Q$ boils down to a univariate quantile estimation problem, so we proceed to examine this first. Assume that every marginal satisfies a log-GW tail limit (i.e., the univariate tail LDP, see Section 2). Let $X_{j, 1: n} \leq \ldots \leq X_{j, n: n}$ be the marginal order statistics derived from the marginal sample $X_{j}^{(1)}, \ldots, X_{j}^{(n)}$. For some intermediate sequence $\left(k_{n}\right)$ and for $n$ large enough that $X_{j, n-k_{n}+1: n}>0$ for $j=$ $1, . ., m$, define the following estimator $\hat{q}_{j, n}$ for $q_{j}$ (compare (3.13)):

$$
\hat{q}_{j, n}(z):= \begin{cases}X_{j,\left\lfloor n\left(1-\mathrm{e}^{-z}\right)\right\rfloor+1: n} & \text { if } z \in\left[0, y_{n}\right] \\ X_{j, n-k_{n}+1: n} \exp \left(\hat{g}_{j, n} h_{\hat{\theta}_{j, n}}\left(z / y_{n}\right)\right) & \text { if } z>y_{n}\end{cases}
$$

with

$$
y_{n}:=\log \left(n / k_{n}\right)
$$

For $z>y_{n}, \hat{q}_{j, n}(z)$ follows a $\log -\mathrm{GW}$ tail with $\hat{\theta}_{j, n}$ and $\hat{g}_{j, n}$ estimators for $\theta_{j}$ and $g_{j}$ in Eq. 3.13, respectively; for other $z$, the empirical quantile is used as estimator. The only assumption we make on the quantile estimator is that the probability-based quantile estimation error $\hat{v}_{j, n}$, defined by

$$
\hat{v}_{j, n}(z):=\frac{\log \left(1-F_{j}\left(\hat{q}_{j, n}(z)\right)\right)}{\log \left(1-F_{j}\left(q_{j}(z)\right)\right)}-1=\frac{q_{j}^{-1} \hat{q}_{j, n}(z)}{z}-1, \quad z \geq 0
$$

satisfies for all $\Lambda>1$ and $j=1, . ., m$ that

$$
\lim _{n \rightarrow \infty} \sup _{\lambda \in[1, \Lambda]}\left|\hat{v}_{j, n}\left(y_{n} \lambda\right)\right|=0 \quad \text { a.s. }
$$

Estimators $\hat{\theta}_{n, j}$ and $\hat{g}_{n, j}$ in Eq. 5.2 satisfying this requirement were considered in de Valk (2016). Let

$$
\hat{Q}_{n}(x):=\left(\hat{q}_{1, n}\left(x_{1}\right), \ldots, \hat{q}_{m, n}\left(x_{m}\right)\right)
$$


for every $x \in[0, \infty)^{m}$. Define as estimator for $Y_{j}^{(i)}:=-\log \left(1-F\left(X_{j}^{(i)}\right)\right)$ :

$$
\hat{Y}_{j, n}^{(i)}:=-\log \left(1-\left(R_{j, n}^{(i)}-\frac{1}{2}\right) / n\right)
$$

with $R_{j, n}^{(i)}:=\sum_{l=1}^{n} \mathbf{1}\left(X_{j}^{(l)} \leq X_{j}^{(i)}\right)$ the marginal rank of $X_{j}^{(i)}$.

For every $n$-tuple of events $\mathcal{C}_{n}:=\left(\mathcal{C}_{n}^{(1)}, \ldots, \mathcal{C}_{n}^{(n)}\right)$ satisfying $\mathcal{C}_{n}^{(i)} \in \mathcal{F}_{n}$ for $i=$ $1, . ., n$, define the "empirical probability" $\hat{p}_{n}\left(\mathcal{C}_{n}\right):=\omega \mapsto \hat{p}_{n}\left(\mathcal{C}_{n}\right)(\omega)$ on $\Omega$ by

$$
\hat{p}_{n}\left(\mathcal{C}_{n}\right)(\omega):=n^{-1} \sum_{i=1}^{n} \mathbf{1}\left(\omega \in \mathcal{C}_{n}^{(i)}\right)
$$

For some $\xi>0$, determine a value of the analogue of $\ell$ in Eq. 5.1 as

$$
\ell_{n}^{+}(B):=\sup \left\{l>0: \hat{p}_{n}\left(\hat{Q}_{n}\left(\hat{Y}_{n} / l\right) \in B\right) \geq\left(k_{n} / n\right)^{\xi}\right\},
$$

with $\sup \{\varnothing\}:=0$ and with $\hat{p}_{n}\left(\hat{Q}_{n}\left(\hat{Y}_{n} / l\right) \in B\right)=n^{-1} \sum_{i=1}^{n} \mathbf{1}\left(\hat{Q}_{n}\left(\hat{Y}_{n}^{(i)} / l\right) \in B\right)$ in accordance with (5.8).

Let $\pi$ denote the probability measure corresponding to $F$. Now consider the following estimator for $\pi(B):=P(X \in B)$ :

$$
\hat{\pi}_{n}^{\mathrm{I}}(B):=\left(k_{n} / n\right)^{\xi / \ell_{n}^{+}(B)} .
$$

If $B_{n, \tau}:=Q(A \tau \log n)$ is substituted for $B$, then under mild restrictions on $A$ and $\left(k_{n}\right)$, this estimator converges in the large deviation sense for all $\tau>0$ :

Theorem 3 Let $X^{(1)}, X^{(2)}$, ... be iid copies of a random vector $X$ on $\mathbb{R}^{m}$ satisfying the conditions of Theorem 1(a), including continuous marginals satisfying log-GW tail limits. For a sequence $\left(k_{n}\right)$ satisfying

$$
0 \leq c^{\prime}:=\liminf _{n \rightarrow \infty} \frac{\log k_{n}}{\log n} \leq \limsup _{n \rightarrow \infty} \frac{\log k_{n}}{\log n}=: c<1,
$$

consider the estimator (5.10) for $P(X \in B)$, with the quantile estimator (5.2) satisfying (5.5) and with $\xi \in\left(0,\left(1-c^{\prime}\right)^{-1}\right)$. Then for $B_{n, \tau}:=Q(A \tau \log n)$, with $A \subset[0, \infty)^{m}$ any Borel set which is a continuity set of I defined by Eqs. 3.2 and 3.11 and satisfies $\inf I(A) \in(0, \infty)$,

$$
\lim _{n \rightarrow \infty} \sup _{\tau \in\left[T^{-1}, T\right]}\left|\frac{\log \hat{\pi}_{n}^{\mathrm{I}}\left(B_{n, \tau}\right)}{\log P\left(X \in B_{n, \tau}\right)}-1\right|=0 \quad \text { a.s. }
$$

for every $T>1$.

The proof can be found in Section 8.3.

Remark 6 By Eq. 3.12, as inf $I\left(A^{o}\right)=\inf I(\bar{A}), P\left(X \in B_{n, \tau}\right)=n^{-\tau \inf I(A)(1+o(1))}$ in Eq. 5.12, so the probability range (1.1) is covered by Theorem 3 .

In practice, computing or approximating (5.9) may not be easy; for example, in engineering applications, it may involve running a complex numerical model for 
every datapoint. Therefore, it would be an advantage to replace $\ell_{n}^{+}(B)$ in Eq. 5.10 by an arbitrary value in some suitable interval. Define for some $\vartheta \in(0, \xi]$

$$
\ell_{n}^{-}(B):=\sup \left\{l>0: \hat{p}_{n}\left(\hat{Q}_{n}\left(\hat{Y}_{n} / l\right) \in B\right) \geq\left(k_{n} / n\right)^{\vartheta}\right\} .
$$

Then $\ell_{n}^{-}(B) \leq \ell_{n}^{+}(B)$. Let $\ell_{n}(B)$ be the result of an algorithm designed to satisfy

$$
\ell_{n}(B) \in\left[\ell_{n}^{-}(B), \ell_{n}^{+}(B)\right] ;
$$

for the present analysis, it is sufficient to assume that $\ell_{n}(B)$ is a random variable satisfying (5.14). Now consider the following generalisation of the estimator (5.10) for $\pi(B):=P(X \in B)$ :

$$
\hat{\pi}_{n}^{\mathrm{II}}(B):=\left(\hat{p}_{n}\left(\hat{Q}_{n}\left(\hat{Y}_{n} / \ell_{n}(B)\right) \in B\right)\right)^{1 / \ell_{n}(B)} .
$$

Theorem 4 For $X^{(1)}, X^{(2)}, \ldots,\left(k_{n}\right)$ and $c^{\prime}$ as in Theorem 3, consider the estimator (5.15) for $P(X \in B)$, with the quantile estimator (5.2) satisfying (5.5) and with $\xi \in\left(0,\left(1-c^{\prime}\right)^{-1}\right)$ and $\vartheta \in(0, \xi]$. Then for $B_{n, \tau}$ as in Theorem 3,

$$
\lim _{n \rightarrow \infty} \sup _{\tau \in\left[T^{-1}, T\right]}\left|\frac{\log \hat{\pi}_{n}^{\mathrm{II}}\left(B_{n, \tau}\right)}{\log P\left(X \in B_{n, \tau}\right)}-1\right|=0 \quad \text { a.s. }
$$

for every $T>1$.

The proof can be found in Section 8.2.

The constraints on $\left(k_{n}\right), \xi$ and $\vartheta$ ensure that for some $\varepsilon \in\left(0, \frac{1}{2}\right)$, eventually, $n^{\varepsilon}<n \hat{p}_{n}\left(\hat{Q}_{n}\left(\hat{Y}_{n} / \ell_{n}\left(B_{n, \tau}\right)\right) \in B_{n, \tau}\right)<n^{1-\varepsilon}$. This does not seem restrictive for applications.

In practice, based on a few trial values of $\ell_{n}(B)$ which give "acceptable" numbers of $n \hat{p}_{n}\left(\hat{Q}_{n}\left(\hat{Y}_{n} / \ell_{n}(B)\right) \in B\right)$, one could check the stability of $\hat{\pi}_{n}^{\mathrm{II}}(B)$ with respect to $n \hat{p}_{n}\left(\hat{Q}_{n}\left(\hat{Y}_{n} / \ell_{n}(B)\right) \in B\right)$.

\section{Numerical examples}

First, we discuss simulations, considering the case of a bivariate normal random vector $U$ with standard normal marginals and correlation coefficient $\rho=0.5$. We are not yet concerned with marginal estimation, so for $X$, we take the random vector with standard exponential marginals obtained from $U$ by marginal transformations; therefore, $X$ equals $Y$ defined by Eq. 3.11 in this case.

As extreme events, we consider halfspaces, i.e., $U \in\left\{x \in \mathbb{R}^{2}: a_{1} x_{1}+a_{2} x_{2}>c\right\}$ for some $a \in \mathbb{R}^{2}$ and $c>0$; their probabilities are easily calculated. In terms of $X$, these events are represented by $X \in B$ with

$$
B=\left\{x \in[0, \infty)^{m}: a_{1} \Phi^{-1}\left(1-\mathrm{e}^{-x_{1}}\right)+a_{2} \Phi^{-1}\left(1-\mathrm{e}^{-x_{2}}\right)>c\right\} .
$$

Experiments were performed with $a_{2}=1$ and with several different values of $a_{1}$, with $c$ in each case chosen to ensure that $P(X \in B)=4 \cdot 10^{-8}$. In all experiments, $n=5000$, and the estimator (5.10) was applied with $\xi=1$ and $k_{n}=20$. 

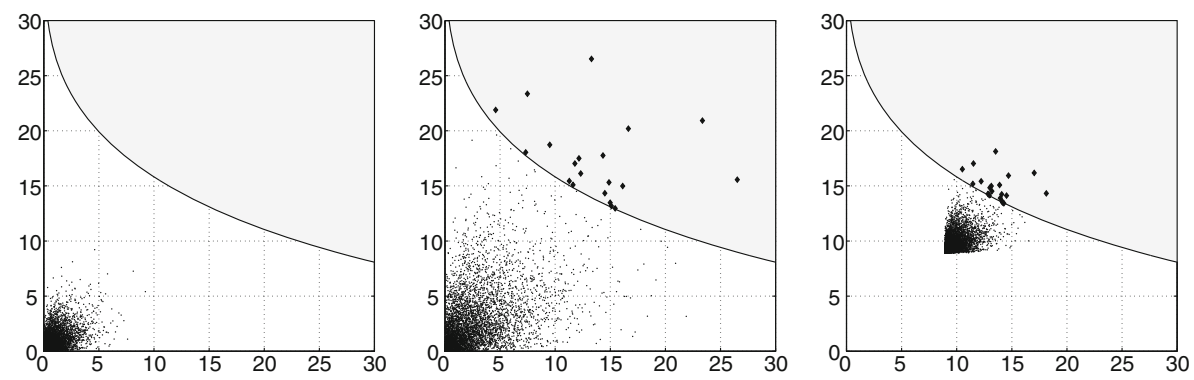

Fig. 3 Simulation with bivariate normal dependence and exponential marginals (see text). From left to right: a $\hat{Y}_{n}$ (dots) and failure event $B$ given by Eq. 6.1 (grey); b $\hat{Y}_{n} / \ell_{n}^{+}(B)$ and failure event; $\mathbf{c}$ the classical analogue $\hat{Y}_{n}+\lambda_{n}(B)$ of (b) (see main text)

Our first case concerns $a_{1}=0.5 ; B$ is shown as a grey patch in Fig. 3. Figure 3a shows $\hat{Y}_{n}$, which has no datapoint in $B$. The stretched data cloud $\hat{Y}_{n} / \ell_{n}^{+}(B)$ is shown in Fig. 3b, with $k_{n}=20$ datapoints in $B ; \ell_{n}^{+}(B)$ equals 0.334 . According to Eq. 5.10, the probability of $B$ is estimated as $(20 / 5000)^{1 / 0.334}=6.6 \cdot 10^{-8}$.

To appreciate how this estimator differs from the classical approach, an estimator similar to Eq. 5.10 but based on the classical multivariate tail limit (1.5) was applied as well: $\hat{\pi}_{n}^{\mathrm{cl}}(B):=\left(k_{n} / n\right) \mathrm{e}^{-\lambda_{n}(B)}$ with $\lambda_{n}(B):=\inf \left\{l>0: \hat{p}_{n}\left(\hat{Y}_{n}+l 1\right) \in\right.$ $\left.B) \geq k_{n} / n\right\}$ with $l=(1,1)$. It is similar to the estimator considered in de Haan and Sinha (1999) and Drees and de Haan (2015) without marginal estimation. Figure $3 \mathrm{c}$ shows $\hat{Y}_{n}+\lambda_{n}(B) l$ with $\lambda_{n}(B)=8.92$; the corresponding probability estimate equals $5.4 \cdot 10^{-7}$. The qualitative difference between Fig. $3 \mathrm{~b}$ and $\mathrm{c}$ is striking.

Figure 4 summarises the results of simulations with $a_{1}=$ $1,0.5,0.1,0,-0.1,-0.5$ and $a_{2}=1$, in each case with 500 realisations: (a) shows the boundaries of the events considered, labelled by $a_{1}$; (b) shows the root mean square errors (RMSE) of the logarithms of the probability estimates, and (c) shows the bias of the logarithms of the probability estimates. For the bivariate normal with
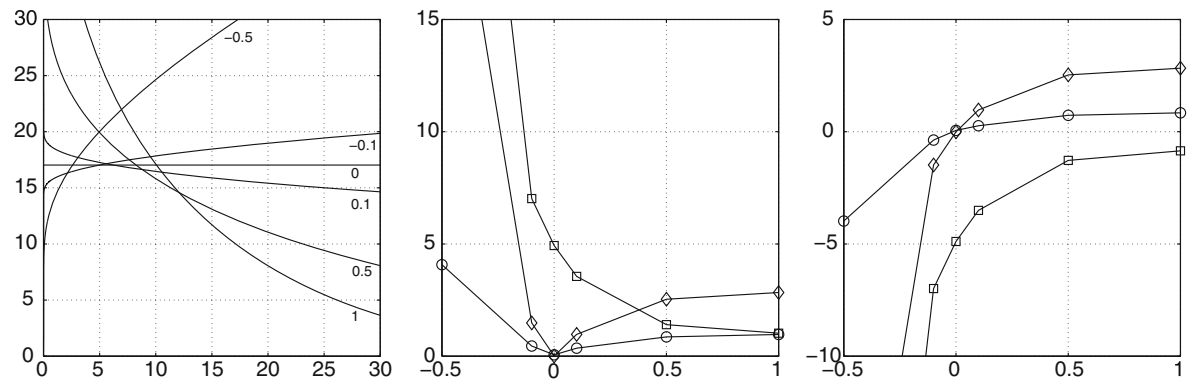

Fig. 4 Simulations with bivariate normal dependence and exponential marginals (see text). From left to right: a boundaries of failure events (6.1) labelled by $a_{1}$, for $a_{2}=1$; b RMSE of the logarithm of probability as function of $a_{1}$ for estimator (5.10) (circles), its classical analogue (diamonds) and its classical analogue accounting for residual tail dependence (squares); $\mathbf{c}$ bias for the estimators as under (b) 
$\rho<1$, the limiting measure in Eq. 1.5 is concentrated on the boundaries, so the classical estimator is not expected to do well in this case. Therefore, in addition, a correction of the classical estimator based on residual tail dependence (4.1) was applied cf. Draisma et al. (2004).

The results in Fig. 4 indicate that the standard deviation is generally small in comparison to the bias, despite the small value of $k_{n}$ used. The two classical estimators perform better or worse depending on the value of $a_{1}$, but the LDP-based estimator (5.10) performs consistently as good as or better than both classical estimators in all cases.

The probability estimator (5.10) can also be applied to estimate the survival function. This makes it possible to compare it to the estimator for the survival function proposed in Wadsworth and Tawn (2013) (Sections 5.1 and 5.2) based on Eq. 4.7 with $\inf I\left(A_{a}\right)$ replaced by $\kappa(a)$, estimated using an approach employing the Hill estimator. With both estimators, the same simulations were carried out as reported in Section 5.3 of Wadsworth and Tawn (2013): for $X$ considered above, estimates of the survival function $F^{c}\left(x_{1}, x_{2}\right)$ were made with $x_{2}=1.5 \log n$ and $x_{1} / x_{2}=0.05,0.10, \ldots, 0.50$. With $n=5000, k_{n}=20,500$ realisations, and $\hat{Y}_{n}$ replaced by the exact $Y$ (see (3.11)) as in Wadsworth and Tawn (2013), the RMSE of the logarithm of probability for Eq. 5.10 was 11-17 \% higher than for the estimator from Wadsworth and Tawn (2013), which performed similarly to an estimator based on the conditional probability approach of Heffernan and Tawn (2004) (see Section 5.3 of Wadsworth and Tawn (2013)). This is an encouraging result for an estimator as simple and widely applicable as (5.10).

The final case is a trial application of the estimator (5.15) to an oceanographic dataset, in order to estimate the mean fraction of time that wave run-up reaches the crest of a fictitious seawall. Figure 5 (upper right) shows simultaneous 3-hourly values of wave period measured at the offshore site YM6 in the North Sea and surge level from the nearby harbour of IJmuiden provided by Rijkswaterstaat, the Netherlands. ${ }^{6}$ The dataset covers 24 years $(n=70128)$. For this trial, a strongly simplified version of a model from TAW (2002) is used to approximate the run-up height of the $2 \%$ highest waves on the seawall from wave period and still water level. The set $B$ of wave period/water level combinations leading to wave run-up exceeding $15 \mathrm{~m}$ is indicated by the grey area in the same figure. In the model, the mean depth at the seawall base is $0 \mathrm{~m}$ and the seawall has a flat smooth 1:4 slope. The RMS wave height at the base is approximated by its upper bound from Ruessink et al. (2003). For the water level, we use surge data, ignoring the astronomical tide. Dependence on wave direction is ignored in marginals and nearshore wave transformation. Because of all these simplifications, estimates obtained do not carry concrete relevance to coastal flood safety.

Quantile estimates for wave period and surge were made using the simple log-GW-based quantile estimator from de Valk (2016): let $\left(k_{2, n}\right)$ be a nondecreasing intermediate sequence satisfying that $\limsup _{n \rightarrow \infty} \log k_{2, n} / \log n<1$ and

\footnotetext{
${ }^{6}$ Wave period is $T_{m_{-1,0}}(\mathrm{~s})$; surge is still water level minus estimated astronomical tide (m).
} 

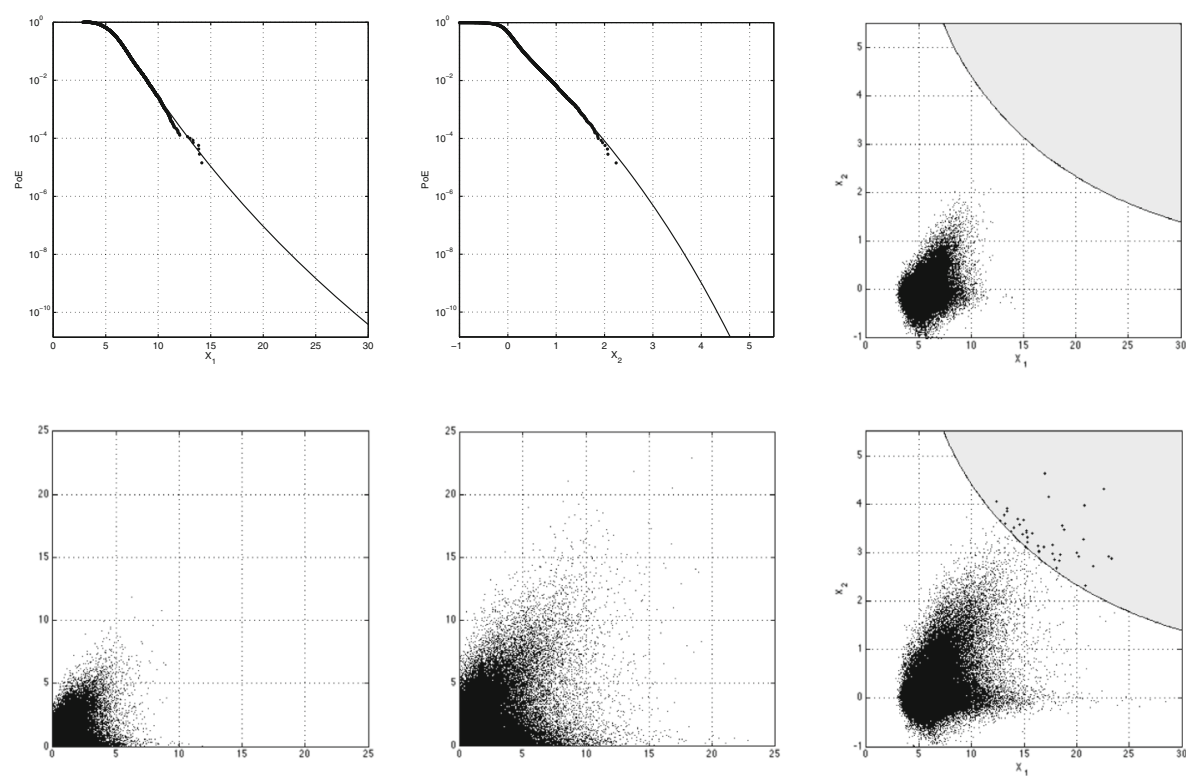

Fig. 5 Marginal log-GW tail estimates for wave period $X_{1}$ (upper left) and surge level $X_{2}$ (upper middle); sample of $X$ and set $B$ corresponding to wave run-up exceeding $15 \mathrm{~m}$ (upper right); $\hat{Y}_{n}$ (lower left); $\hat{Y}_{n} / \ell_{n}(B)$ (lower middle); $\hat{Q}_{n}\left(\hat{Y}_{n} / \ell_{n}(B)\right)$ (lower right); fat dots indicating points with run-up exceeding $15 \mathrm{~m}$

$\lim _{n \rightarrow \infty} k_{2, n} / \log _{2} n=\infty$ (with $\log _{2}$ the iterated logarithm), fix some $\iota>1$, and define

$$
k_{i, n}:=\left\lfloor\left(n / k_{2, n}\right)^{-l^{i-2}} n\right\rfloor \quad \text { for } i \in\{0,1\} .
$$

Taking $k_{n}=k_{0, n}$ in Eq. 5.2, and

$$
\hat{\theta}_{n, j}:=\frac{\log _{2} \frac{X_{j, n-k_{2, n}+1: n}}{X_{j, n-k_{1, n}+1: n}}-\log _{2} \frac{X_{j, n-k_{1, n}+1: n}}{X_{j, n-k_{0, n}+1: n}}}{\log \iota} \quad \text { and } \quad \hat{g}_{n, j}:=\frac{\log \frac{X_{j, n-k_{1, n}+1: n}}{X_{j, n-k_{0, n}+1: n}}}{h_{\hat{\theta}_{n, j}}(\iota)}
$$

Equation 5.5 is ensured by Theorem 4 of de Valk (2016). Like the Pickands (1975) estimator for the extreme value index, the estimator $\hat{\theta}_{n}$ is based on only three order statistics. However, its behaviour is entirely different, because the spacings between $\left(k_{0, n}\right),\left(k_{1, n}\right)$ and $\left(k_{2, n}\right)$ are different.

Quantile estimates (5.2) with (6.2)-(6.3), $\iota=2$ and $k_{n}=5009$ are shown in the upper left and middle panels of Fig. 5. The lower left panel shows $\hat{Y}_{n}$, the lower middle panel shows $\hat{Y}_{n} / \ell_{n}(B)$ with $\ell_{n}(B)=1 / 2.13 ; \hat{Q}_{n}\left(\hat{Y}_{n} / \ell_{n}(B)\right)$ is shown in the lower right panel. In this case, $\hat{p}_{n}\left(\hat{Q}_{n}\left(\hat{Y}_{n} / \ell_{n}(B)\right) \in B\right)=41 / n=5.85 \cdot 10^{-4}$, so for the estimator $(5.15), \hat{\pi}_{n}^{\mathrm{II}}(B)=(41 / n)^{2.13}=1.3 \cdot 10^{-7}$, about 11 hours per 10,000 years.

Contrary to the assumptions made earlier, the 3-hourly surge and wave period are serially dependent. Since we are estimating a fraction of time, serial dependence does not need to invalidate the estimate; its principal effect is that the estimate is less precise than it would have been if the process were iid. Imposing a minimum 
separation of 24 hours between storm events, the 41 datapoints moved into $B$ in Fig. 5 (lower right) represent 18 distinct events, giving a mean duration per event of 6.8 hours. Using this value, the estimate $\hat{\pi}_{n}^{\mathrm{II}}(B)$ can be converted to an estimate of the frequency of wave run-up exceeding $15 \mathrm{~m}$; its value is $1.7 \cdot 10^{-4}$ per year. Evidently, this unconventional, but intuitively appealing variation of the peaks-over-threshold approach would need formal underpinning by a model of serial dependence in order to be taken seriously.

\section{Discussion}

Like similar methods in the classical setting (e.g. de Haan and Sinha (1999), Drees and de Haan (2015), Draisma et al. (2004)), the estimators (5.10) and (5.15) exploit homogeneity of a function describing tail dependence; in this case, homogeneity (3.4) of the rate function $I$. This offers the advantage that no explicit estimate of $I$ is required. However, in certain situations, there may be good reasons to estimate $I$, such as if for a given random vector $X$, probabilities need to be estimated for multiple sets in a consistent and reproducible manner. Therefore, estimation of $I$ remains a topic deserving elaboration.

The limitation of $A$ to continuity sets of $I$ in Theorems 3 and 4 is less restrictive than it may seem, since the homogeneity of $I$ makes continuity sets rather common, as noted in Section 3. The other conditions on $A$ are weak.

To prove convergence of the estimators under such weak conditions, local uniformity in $d$ of convergence in Eq. 8.5 is employed, which is derived from uniformity in $d$ of convergence in (8.12). The latter also ensures local uniformity in $\lambda$ of convergence in Eq. 8.5, and therefore local uniformity in $\tau$ of convergence of the estimators in Eqs. 5.12 and 5.16. In practice, this means that if such an estimator applied to a given dataset produces a fair estimate of $P\left(X \in B_{0}\right)$ for some $B_{0} \subset \mathbb{R}^{m}$, then it may also be applied with confidence to the same dataset to estimate the probability of $B_{1} \subset \mathbb{R}^{m}$ such that $P\left(X \in B_{1}\right) \geq P\left(X \in B_{0}\right)^{\tau}$ for $\tau>1$ not too large, e.g. $\tau=2$. If $P\left(X \in B_{0}\right) \ll 1$, e.g. $P\left(X \in B_{0}\right)=0.01$, this amounts to extrapolation over several additional orders of magnitude in probability. How far one can extrapolate in practice will depend on the rates of convergence to the marginal log-GW tail limits and in Eq. 3.1, which will differ from case to case.

Convergence of log-probability ratios as in Eqs. 5.12 and 5.16 is typical for the probability range (1.1). A stronger notion of convergence might be desirable, but would require restrictive additional assumptions which would be hard to justify in applications. Rather, it is recommended to diagnose bias in estimates and take this into account in estimates of uncertainty. For this reason, modelling of bias and rate of convergence deserves further study.

Deriving asymptotic error distributions will require additional assumptions beyond those for Theorems 3 and 4 and methods quite different from those employed in the present article. Because it is complex (see e.g. Drees and de Haan (2015) for a comparable problem), this important topic needs to be left for a follow-up study as well.

The theory is readily extended from events involving a high value of at least one of the variables to events extreme "in any direction", by replacing the exponential 
distribution as standard marginal by the Laplace distribution cf. Keef et al. (2013). Other choices of standard marginal are also possible, with minor adaptations to theory and estimator.

Furthermore, the main results of this article can be generalised straightforwardly from a random vector in $\mathbb{R}^{m}$ to a random element of $\mathcal{C}_{b}(K)$, the continuous functions on a compact metric space $K$. Classical multivariate extreme value theory and estimation have been generalised to this setting earlier; see e.g. de Haan and Lin (2001), Part III of de Haan and Ferreira (2006), Einmahl and Lin (2006) and Ferreira and de Haan (2014). For the theory presented here, the main difference between the $\mathbb{R}^{m}$ setting and the $\mathcal{C}_{b}(K)$ setting is that in the latter, exponential tightness of $\{P(Y / y \in \cdot), y>0\}$ no longer follows from the exponential marginals; it is an independent assumption. In loose terms, it entails that all but an exponentially small probability mass is concentrated on an equicontinuous set of functions in $\mathcal{C}_{b}(K)$ (see e.g. Dembo and Zeitouni (1998)).

\section{Proofs and lemmas}

\subsection{Proof of Theorem 1 and Corollary 1}

Convergence in Eq. 2.6 is locally uniform in $\lambda$ (e.g. de Haan and Ferreira (2006), B.1.4 and B.2.9), so for all $\Lambda>1$,

$$
\lim _{y \rightarrow \infty} \sup _{\lambda \in\left[\Lambda^{-1}, \Lambda\right]} \max _{j=1, \ldots, m}\left|h_{\theta j}^{-1}\left(\frac{\log q_{j}(y \lambda)-\log q_{j}(y)}{g_{j}(y)}\right)-\lambda\right|=0 .
$$

For every $y>0, \tilde{Q}_{y}$ is injective, so we can define the random vector

$$
\tilde{Y}_{y}:=\tilde{Q}_{y}^{-1}(X)=\tilde{Q}_{y}^{-1} Q(Y) \quad \text { a.s. }
$$

with $Y$ defined by Eq. 3.11. By Eq. 8.1, there exists almost surely for every $\Lambda>1$ and $\delta>0$ some $y_{\Lambda, \delta}>0$ such that for all $y \geq y_{\Lambda, \delta},\left\|\tilde{Y}_{y}-Y\right\|_{\infty}>\delta y$ implies $\|Y\|_{\infty}>\Lambda y$. Therefore, by Eq. 3.5, since $\Lambda>1$ is arbitrary, for all $\delta>0$,

$$
\lim _{y \rightarrow \infty} \frac{1}{y} \log P\left(\left\|\tilde{Y}_{y}-Y\right\|_{\infty}>\delta y\right)=-\infty .
$$

By Proposition 3, the distribution functions of $\{Y / y, y>0\}$ satisfy the LDP (3.1) with good rate function $I$, so Eq. 8.3 implies the same for the distribution functions of $\left\{\tilde{Y}_{y} / y, y>0\right\}$; see Theorem 4.2.13 of Dembo and Zeitouni (1998). Therefore, Eq. 3.15 follows from (8.2). To prove (b), note that by Eqs. 3.15 and 3.7,

$$
\lim _{y \rightarrow \infty} \frac{1}{y} \log \left(1-F_{j}\left(q_{j}(y) \mathrm{e}^{g_{j}(y) h_{\theta_{j}}(\lambda)}\right)\right)=-\lambda
$$

for all $\lambda \geq 1$ and $j=1, . ., m$, so Eq. 2.6 holds with $q=q_{j}, g=g_{j}$ and $\theta=\theta_{j}$ for $j=1, . ., m$. As in the proof of (a), this implies (8.3). Moreover, Eq. 3.7 implies (3.3), so $I$ is a good rate function. An application of Theorem 4.2.13 of Dembo and Zeitouni (1998) completes the proof of the theorem. 
For the Corollary, note that for $A \subset[1, \infty)^{m}, P\left(X \in \tilde{Q}_{y}(y A)\right)$ in Eq. 3.15 is equal to

$$
P\left(\left(\frac{\log X_{1}-\log q_{1}(y)}{g_{1}(y)}, . ., \frac{\log X_{m}-\log q_{m}(y)}{g_{m}(y)}\right) \in H_{\theta}(A)\right)
$$

by Eq. 3.13. Therefore, by the contraction principle (see Theorem 4.2.1 in Dembo and Zeitouni (1998)), Eq. 3.16 follows from (3.15).

\subsection{Proof of Theorem 4}

For convenience, the following shorthand notation will be used:

$$
\hat{\mu}_{n, l}(A):=\hat{p}_{n}\left(\hat{Q}_{n}\left(\hat{Y}_{n} / l\right) \in Q\left(y_{n} A\right)\right),
$$

and $l_{n}(A):=\ell_{n}\left(Q\left(y_{n} A\right)\right), l_{n}^{+}(A):=\ell_{n}^{+}\left(Q\left(y_{n} A\right)\right), l_{n}^{-}(A):=\ell_{n}^{-}\left(Q\left(y_{n} A\right)\right)$.

By Proposition 3, $Y$ defined by Eq. 3.11 satisfies the LDP (3.1) with good rate function $I$. Noting that $\xi \in\left(0,\left(1-c^{\prime}\right)^{-1}\right)$ with $c^{\prime}$ as in Eq. 5.11, take any $\Delta \in$ $\left(\xi / \inf I(A),\left(1-c^{\prime}\right)^{-1} / \inf I(A)\right)$. Fixing an arbitrary $\Lambda>1$, then by Lemma 4 , for every $\delta \in(0, \Delta)($ see $(8.4))$,

$$
\lim _{n \rightarrow \infty} \sup _{\lambda \in\left[\Lambda^{-1}, \Lambda\right], d \in[\delta, \Delta]}\left|y_{n}^{-1} \log \hat{\mu}_{n, d / \lambda}(A \lambda)+d \inf I(A)\right|=0 \quad \text { a.s. }
$$

and

$$
\limsup _{n \rightarrow \infty} \sup _{\lambda \in\left[\Lambda^{-1}, \Lambda\right], d>\Delta} y_{n}^{-1} \log \hat{\mu}_{n, d / \lambda}(A \lambda) \leq-\Delta \inf I(A)<-\zeta \quad \text { a.s. }
$$

Choosing $\delta<\vartheta / \inf I(A)$, since $\Delta>\xi / \inf I(A)$, we observe that

$$
d \inf I(A)\left\{\begin{array}{l}
\leq \xi \text { if } d \in[\delta, \xi / \inf I(A)] \subset[\delta, \Delta] \\
>\xi \text { if } d \in(\xi / \inf I(A), \Delta] \subset[\delta, \Delta]
\end{array}\right.
$$

in Eq. 8.5. Therefore, with Eq. 8.6, using Eq. 5.9,

$$
\lim _{n \rightarrow \infty} \sup _{\lambda \in\left[\Lambda^{-1}, \Lambda\right]}\left|\lambda l_{n}^{+}(A \lambda)-\xi / \inf I(A)\right|=0 \quad \text { a.s. }
$$

and similarly, using Eq. 5.13, we find that

$$
\lim _{n \rightarrow \infty} \sup _{\lambda \in\left[\Lambda^{-1}, \Lambda\right]}\left|\lambda l_{n}^{-}(A \lambda)-\vartheta / \inf I(A)\right|=0 \quad \text { a.s. }
$$

By Eqs. 8.7, 8.8, 5.14 and 8.5,

$$
\lim _{n \rightarrow \infty} \sup _{\lambda \in\left[\Lambda^{-1}, \Lambda\right]}\left|y_{n}^{-1} l_{n}^{-1}(A \lambda) \log \hat{\mu}_{n, l_{n}(A \lambda)}(A \lambda)+\lambda \inf I(A)\right|=0 \quad \text { a.s. }
$$

or equivalently, by Eq. 5.15,

$$
\lim _{n \rightarrow \infty} \sup _{\lambda \in\left[\Lambda^{-1}, \Lambda\right]}\left|y_{n}^{-1} \log \hat{\pi}_{n}^{\mathrm{II}}\left(Q\left(y_{n} A \lambda\right)\right)+\lambda \inf I(A)\right|=0 \quad \text { a.s. }
$$


Since Eq. 3.12 holds with $\inf I\left(A^{o}\right)=\inf I(\bar{A})$ and $\inf I(A)>0$, by Eqs. 3.4 and 8.10 ,

$$
\lim _{n \rightarrow \infty} \sup _{\lambda \in\left[\Lambda^{-1}, \Lambda\right]}\left|\frac{\log \hat{\pi}_{n}^{\mathrm{II}}\left(Q\left(y_{n} A \lambda\right)\right)}{\log P\left(X \in Q\left(y_{n} A \lambda\right)\right)}-1\right|=0 \quad \text { a.s., }
$$

and Eq. 5.16 follows from Eq. 5.11, since $\Lambda>1$ is arbitrary.

\subsection{Proof of Theorem 3}

Following the proof of Theorem 4 in Section 8.2, Eqs. 8.7 and 5.10 yield

$$
\lim _{n \rightarrow \infty} \sup _{\lambda \in\left[\Lambda^{-1}, \Lambda\right]}\left|y_{n}^{-1} \log \hat{\pi}_{n}^{\mathrm{I}}\left(Q\left(y_{n} A \lambda\right)\right)+\lambda \inf I(A)\right|=0,
$$

and the result Eq. 5.12 follows as in the proof of Theorem 4.

\subsection{Lemmas}

Lemma 1 Let $Y$ be a random vector in $[0, \infty)^{m}$ with standard exponential marginals satisfying the LDP (3.1) with good rate function $I$, and $Y^{(1)}, Y^{(2)}, \ldots$ a sequence of iid copies of $Y$. Let the Borel set $A \subset[0, \infty)^{m}$ be a continuity set of I satisfying $\inf I(A) \in(0, \infty)$. If $\left(y_{n}>0\right)$ and $\Delta>0$ satisfy $\lim _{n \rightarrow \infty} y_{n}=\infty$ and

$$
\Delta<\liminf _{n \rightarrow \infty} \frac{\log n}{y_{n} \inf I(A)}<\infty,
$$

then with $\hat{p}_{n}$ defined by Eq. 5.8, for all $\delta \in(0, \Delta)$,

$$
\lim _{n \rightarrow \infty} \sup _{d \in[\delta, \Delta]}\left|y_{n}^{-1} \log \hat{p}_{n}\left(Y \in d A y_{n}\right)+d \inf I(A)\right| \rightarrow 0 \quad \text { a.s. }
$$

and

$$
\limsup _{n \rightarrow \infty} \sup _{d>\Delta} y_{n}^{-1} \log \hat{p}_{n}\left(Y \in d A y_{n}\right) \leq-\Delta \inf I(A) \quad \text { a.s. }
$$

Proof Let $\mathcal{A}:=\cup_{\lambda \geq 1}(\lambda A)$; by Eq. 3.4, $\mathcal{A}$ is a continuity set of $I$ satisfying $\inf I(\mathcal{A})=\inf I(A)<\infty$. Define the random variable

$$
v:=\inf \{w>0: Y w \in \mathcal{A}\}
$$

with $\inf \{\emptyset\}:=\infty$, and let $G$ be its distribution function. Since $\cup_{\lambda \geq 1}(\mathcal{A} \lambda) \subset \mathcal{A}$, $Y \in \mathcal{A}^{o} z \Rightarrow v \leq z^{-1} \Rightarrow Y \in \overline{\mathcal{A}} z$ for every $z>0$, so by Eqs. 3.1 and 3.4 , for all $w>0$,

$$
\lim _{y \rightarrow \infty} y^{-1} \log G(w / y)=-w^{-1} \inf I(\mathcal{A}) .
$$

Therefore, since $\inf I(\mathcal{A}) \in(0, \infty),-\log G(1 / \mathrm{Id}) \in R V_{\{1\}}$, so by Bingham et al. (1987) (Theorem 1.5.2) and Eq. 8.15 again, for every $a>0$,

$$
\lim _{y \rightarrow \infty} \sup _{w \geq a}\left|y^{-1} \log G(w / y)+w^{-1} \inf I(\mathcal{A})\right|=0 .
$$

By Eq. 8.15, there is for every $\varepsilon>0$ an $n_{\varepsilon} \in \mathbb{N}$ such that for all $n \geq n_{\varepsilon}$,

$$
n G\left(a / y_{n}\right) \geq \mathrm{e}^{\log n-\left(\varepsilon+a^{-1} \inf I(\mathcal{A})\right) y_{n}} .
$$


Taking $a=1 / \Delta$, then by Eq. 8.11, $\varepsilon>0$ can be chosen small enough that the exponent in Eq. 8.17 eventually exceeds $\varepsilon \log n$. Therefore,

$$
\lim _{n \rightarrow \infty} n G\left(a / y_{n}\right) / \log n=\infty .
$$

With $G^{-1}$ the left-continuous inverse of $G$, almost surely $v^{(i)}=G^{-1}\left(\mathcal{U}^{(i)}\right)$ for all $i \in \mathbb{N}$, with $\mathcal{U}^{(1)}, \mathcal{U}^{(2)}, \ldots$ independent and uniformly distributed on $(0,1)$, so almost surely (see def. (5.8)), $\hat{p}_{n}\left(v \leq w / y_{n}\right)=\hat{p}_{n}\left(\mathcal{U} \leq G\left(w / y_{n}\right)\right)$ for all $n \in \mathbb{N}$ and all $w \geq a$. Therefore, by Wellner (1978) (Corollary 1) and (8.18),

$$
\lim _{n \rightarrow \infty} \sup _{w \geq a}\left|\log \hat{p}_{n}\left(v \leq w / y_{n}\right)-\log G\left(w / y_{n}\right)\right|=0 \quad \text { a.s. },
$$

and since $v \leq w / y_{n} \Rightarrow Y \in \mathcal{A} y_{n} /(w l) \Rightarrow v \leq w l / y_{n}$ for all $l>1$ and $w>0$, using Eqs. 8.16 and 3.4 , as $a=1 / \Delta$,

$$
\lim _{n \rightarrow \infty} \sup _{d \in(0, \Delta]}\left|y_{n}^{-1} \log \hat{p}_{n}\left(Y \in d \mathcal{A} y_{n}\right)+d \inf I(\mathcal{A})\right|=0 \quad \text { a.s. }
$$

Therefore, as $A \subset \mathcal{A}$ and $\inf I(\mathcal{A})=\inf I(A)$,

$$
\limsup _{n \rightarrow \infty} \sup _{d \in(0, \Delta]} y_{n}^{-1} \log \hat{p}_{n}\left(Y \in d A y_{n}\right)+d \inf I(A) \leq 0 \quad \text { a.s. }
$$

$A$ is a continuity set of $I$ and $I$ satisfies (3.4), so there is for every $\varepsilon>0$ a point $x_{\varepsilon} \in A^{o}$ such that $I\left(x_{\varepsilon}\right)<\inf I(A)+\varepsilon$. Let $\varepsilon>0$ and $\eta>1$ be such that $\Delta \eta(\inf I(A)+\varepsilon)<\liminf _{n \rightarrow \infty} y_{n}^{-1} \log n$ (see (8.11)). Then for $\eta$ sufficiently close to 1 , an open set $\mathcal{B} \subset[0, \infty)^{m}$ can be constructed such that

$$
\begin{gathered}
\cup_{\lambda \geq 1}(\lambda \mathcal{B}) \subset \mathcal{B}, \quad x_{\varepsilon} \in \mathcal{B} \backslash(\mathcal{B} \eta) \subset A^{o}, \quad \text { and } \\
\quad \inf I\left(\mathcal{B}^{o}\right)=\inf I(\overline{\mathcal{B}}) \in\left(\inf I(A), I\left(x_{\varepsilon}\right)\right]
\end{gathered}
$$

as follows. The first two requirements on $\mathcal{B}$ are satisfied by $\mathcal{B}^{\prime}=\cup_{\lambda \geq 1}(\lambda U)$ for some sufficiently small neighbourhood $U \subset A^{o}$ of $x_{\varepsilon}$, with $\eta>1$ close enough to 1 . If $\mathcal{B}^{\prime}$ is a continuity set of $I$, then set $\mathcal{B}=\mathcal{B}^{\prime}$. Else, consider the function $f$ : $[0, \infty)^{m} \times[0,1] \rightarrow[0, \infty)^{m}$ defined by $f(y, a):=a y+(1-a)\left(\|y\|_{\infty} /\left\|x_{\varepsilon}\right\|_{\infty}\right) x_{\varepsilon}$. It satisfies $f\left(\mathcal{B}^{\prime}, 1\right)=\mathcal{B}^{\prime}, f\left(\mathcal{B}^{\prime}, 0\right)=\mathcal{B}^{\prime} \cap \cup_{\lambda>0}\left(\lambda x_{\varepsilon}\right)$, and $f\left(\mathcal{B}^{\prime}, a\right) \subset f\left(\mathcal{B}^{\prime}, a^{\prime}\right)$ if $a \leq a^{\prime}$. Therefore, $a \mapsto \inf I\left(f\left(\mathcal{B}^{\prime}, a\right)\right)$ is non-increasing, so with $\alpha$ any of its continuity points in $(0,1), \mathcal{B}=f\left(\mathcal{B}^{\prime}, \alpha\right)$ is a continuity set of $I$ and satisfies (8.22). By Eq. 8.22,

$$
\begin{aligned}
& \hat{p}_{n}\left(Y \in d A y_{n}\right) \geq \hat{p}_{n}\left(Y \in d \mathcal{B} y_{n}\right)-\hat{p}_{n}\left(Y \in d \eta \mathcal{B} y_{n}\right) \\
= & \hat{p}_{n}\left(Y \in d \mathcal{B} y_{n}\right)\left(1-\mathrm{e}^{\log \hat{p}_{n}\left(Y \in d \eta \mathcal{B} y_{n}\right)-\log \hat{p}_{n}\left(Y \in d \mathcal{B} y_{n}\right)}\right),
\end{aligned}
$$

and furthermore, Eq. 8.20 continues to hold after substituting $\mathcal{B}$ or $\mathcal{B} \eta$ for $\mathcal{A}$. Therefore, by Eq. 3.4, for every $\delta \in(0, \Delta)$ almost surely, the right-hand side of Eq. 8.23 is $\hat{p}_{n}\left(Y \in d \mathcal{B} y_{n}\right)(1+o(1))$ uniformly in $d \in[\delta, \Delta]$ and furthermore, using Eq. 8.22,

$$
\liminf _{n \rightarrow \infty} \inf _{d \in[\delta, \Delta]} y_{n}^{-1} \log \hat{p}_{n}\left(Y \in d A y_{n}\right)+d I\left(x_{\varepsilon}\right) \geq 0 \quad \text { a.s. }
$$

Now Eq. 8.12 follows from Eqs. 8.21 and 8.24, because $I\left(x_{\varepsilon}\right)<\inf I(A)+\varepsilon$, and $\varepsilon>0$ can be chosen arbitrarily close to 0 . Finally, by Eq. 8.20, as $\cup_{\lambda \geq 1}(\mathcal{A} \lambda) \subset \mathcal{A}$,

$$
\limsup _{n \rightarrow \infty} \sup _{d>\Delta} y_{n}^{-1} \log \hat{p}_{n}\left(Y \in d \mathcal{A} y_{n}\right) \leq-\Delta \inf I(\mathcal{A}) \quad \text { a.s. }
$$


and because $A \subset \mathcal{A}$ and $\inf I(\mathcal{A})=\inf I(A),(8.13)$ follows.

Lemma 2 Let $Y$ be a random vector on $[0, \infty)^{m}$ with standard exponential marginals and $Y^{(1)}, Y^{(2)}, \ldots$ a sequence of iid copies of $Y$. Define

$$
\hat{Y}_{n}^{(i)}:=\left(\hat{Y}_{1, n}^{(i)}, . ., \hat{Y}_{m, n}^{(i)}\right)
$$

for $i=1, . ., n$, with

$$
\hat{Y}_{j, n}^{(i)}:=-\log \left(1-\left(R_{j, n}^{(i)}-\frac{1}{2}\right) / n\right)
$$

and

$$
R_{j, n}^{(i)}:=\sum_{l=1}^{n} \mathbf{1}\left(Y_{j}^{(l)} \leq Y_{j}^{(i)}\right) .
$$

For $\left(y_{n}>0\right)$ satisfying $\liminf _{n \rightarrow \infty} y_{n} / \log n>0$,

$$
\sup _{\varepsilon>0} \limsup _{n \rightarrow \infty} y_{n}^{-1} \log \hat{p}_{n}\left(\left\|\hat{Y}_{n}-Y\right\|_{\infty}>y_{n} \varepsilon\right)=-\infty \quad \text { a.s. }
$$

Proof Since $\hat{p}_{n}\left(\left\|\hat{Y}_{n}-Y\right\|_{\infty}>y_{n} \varepsilon\right) \leq \sum_{j=1}^{m} \hat{p}_{n}\left(\left|\hat{Y}_{j, n}-Y_{j}\right|>y_{n} \varepsilon\right)$, it is sufficient to prove (8.27) for the univariate case.

Let $\mathcal{U}^{(i)}:=\exp \left(-Y^{(i)}\right)$, and let $\Gamma_{n}$ and $\Gamma_{n}^{-1}$ denote the empirical distribution function and the empirical quantile function of $\mathcal{U}^{(1)}, . ., \mathcal{U}^{(n)}$, which are independent and uniformly distributed in $(0,1)$. By Theorem 2 of Shorack and Wellner (1978), as $\sup _{t \in[0,1]} t^{-1} \Gamma_{n}(t)=\sup _{t \in[1 / n, 1]} t / \Gamma_{n}^{-1}(t)$,

$$
\limsup _{n \rightarrow \infty} \sup _{t \in[1 / n, 1]} \log \left(t / \Gamma_{n}^{-1}(t)\right) / \log _{2} n=1 \quad \text { a.s. }
$$

Similarly, as $\sup _{t \in\left[\mathcal{U}_{1: n}, 1\right]} t / \Gamma_{n}(t)=1 \vee \sup _{t \in[1 / n, 1]} t^{-1} \Gamma_{n}^{-1}(t)$, by Theorem 3 of Shorack and Wellner (1978),

$$
\limsup _{n \rightarrow \infty} \sup _{t \in[1 / n, 1]} t^{-1} \Gamma_{n}^{-1}(t) / \log _{2} n=1 \quad \text { a.s. }
$$

so

$$
\liminf _{n \rightarrow \infty} \inf _{t \in[1 / n, 1]} \log \left(t / \Gamma_{n}^{-1}(t)\right) / \log _{2} n=0 \quad \text { a.s. }
$$

Since $Y_{n-i+1: n}=-\log \Gamma_{n}^{-1}(i / n), \hat{Y}_{n-i+1: n}=-\log \left(\left(i-\frac{1}{2}\right) / n\right)$, and $y_{n} / \log _{2} n \rightarrow \infty$, Eqs. 8.28 and 8.29 imply

$$
\max _{i=1, \ldots, n}\left|Y_{n-i+1: n}-\hat{Y}_{n-i+1: n}\right| / y_{n} \rightarrow 0 \quad \text { a.s. }
$$

As a consequence, there is almost surely for every $\varepsilon>0$ an $n_{\varepsilon} \in \mathbb{N}$ such that for all $n \geq n_{\varepsilon}, \hat{p}_{n}\left(\left|Y-\hat{Y}_{n}\right|>y_{n} \varepsilon\right)=0$ and therefore $y_{n}^{-1} \log \hat{p}_{n}\left(\left|Y-\hat{Y}_{n}\right|>y_{n} \varepsilon\right)=-\infty$, implying the univariate case of Eq. 8.27.

Lemma 3 Let $X$ be a random vector on $\mathbb{R}^{m}$ having continuous marginals satisfying log-GW tail limits, and let $X^{(1)}, X^{(2)}, \ldots$ be a sequence of iid copies of $X$. With $Q$, $\hat{Q}_{n}$ and $\hat{Y}_{n}$ defined by Eqs. 3.10, 5.6 and 5.7, let $\left(k_{n}\right)$ satisfy (5.11) and $\hat{q}_{j, n}$ defined 
by Eq. 5.2 satisfy (5.5) for $j=1, \ldots, m$, with $y_{n}$ defined by Eq. 5.3. Then for every $\delta>0$ and $\varepsilon>0$,

$$
\lim _{n \rightarrow \infty} \sup _{l \geq \delta} y_{n}^{-1} \log \hat{p}_{n}\left(\left\|Q^{-1} \hat{Q}_{n}\left(\hat{Y}_{n} l^{-1}\right)-\hat{Y}_{n} l^{-1}\right\|_{\infty}>y_{n} \varepsilon\right)=-\infty \text { a.s. }
$$

Proof Fix $\varepsilon>0$ and $\delta>0$. As in Lemma 2, we only need to prove (8.31) for the univariate case, so we proceed with this. Note that Eq. 8.31 holds if an $n_{\delta, \varepsilon} \in \mathbb{N}$ exists such that (suppressing the labels of vector components in the univariate case)

$$
\sup _{l \geq \delta} \max _{j=1, \ldots, n}\left|q^{-1} \hat{q}_{n}\left(\hat{Y}_{j: n} l^{-1}\right)-\hat{Y}_{j: n} l^{-1}\right| \leq y_{n} \varepsilon \quad \text { for all } n \geq n_{\delta, \varepsilon} .
$$

Fixing $\Lambda>\max \left(1, \delta^{-1}\right) /(1-c) \geq \max \left(1, \delta^{-1}\right) \lim \sup _{n \rightarrow \infty} \log (2 n) / y_{n}$ with $c$ as in Eqs. 5.11, 8.32 holds if

$$
\sup _{z \in\left[0, y_{n} \Lambda\right]}\left|q^{-1} \hat{q}_{n}(z)-z\right| \leq y_{n} \varepsilon \quad \text { for all } n \geq n_{\delta, \varepsilon} .
$$

Such an $n_{\delta, \varepsilon} \in \mathbb{N}$ exists if $\hat{v}_{n}$ defined by Eq. 5.4 satisfies

$$
\sup _{z \in\left[y_{n}, y_{n} \Lambda\right]}\left|\hat{v}_{n}(z)\right| \rightarrow 0
$$

and also,

$$
\sup _{z \in\left[0, y_{n}\right]}\left|q^{-1} \hat{q}_{n}(z)-z\right| / y_{n}=\sup _{t \in\left[\mathrm{e}^{-y_{n}}, 1\right]}\left|\log \left(t / \Gamma_{n}^{-1}(t)\right)\right| / y_{n} \rightarrow 0,
$$

with $\mathcal{U}^{(1)}, . ., \mathcal{U}^{(n)}$ as in the proof of Lemma 2 (note that for all $z \in\left[0, y_{n}\right], q^{-1} \hat{q}_{n}(z)$ $\left.=q^{-1}\left(X_{\left\lfloor n\left(1-\mathrm{e}^{-z}\right)\right\rfloor+1: n}\right)=Y_{\left\lfloor n\left(1-\mathrm{e}^{-z}\right)\right\rfloor+1: n}=-\log \Gamma_{n}^{-1}\left(\mathrm{e}^{-z}\right)\right)$. As in the proof of Lemma 2, Eqs. 8.28 and 8.29 hold. Therefore, since the upper bound in Eq. 5.11 implies that $\liminf _{n \rightarrow \infty} y_{n} / \log n>0$, Eq. 8.35 holds almost surely. Furthermore, by Eq. 5.5, (8.34) holds almost surely. This proves the univariate case.

Lemma 4 Let the random vector $X$ on $\mathbb{R}^{m}$ have continuous marginals satisfying log$G W$ tail limits and let $Y$ defined by Eq. 3.11 satisfy the LDP (3.1) with good rate function I. Let $X^{(1)}, X^{(2)}, \ldots$ be a sequence of iid copies of $X$. Let $\left(k_{n}\right)$ satisfy (5.11) and let the quantile estimator $\hat{q}_{j, n}$ given by Eq. 5.2 satisfy (5.5). Let the Borel set $A \subset[0, \infty)^{m}$ be a continuity set of I satisfying $\inf I(A) \in(0, \infty)$. Then $\hat{\mu}$ defined by Eq. 8.4 satisfies for every $\Lambda>1$ and every

$$
\Delta \in\left(0, \frac{1}{\left(1-c^{\prime}\right) \inf I(A)}\right)
$$

and $\delta \in(0, \Delta)$ :

$$
\lim _{n \rightarrow \infty} \sup _{\lambda \in\left[\Lambda^{-1}, \Lambda\right], d \in[\delta, \Delta]}\left|y_{n}^{-1} \log \hat{\mu}_{n, d / \lambda}(A \lambda)+d \inf I(A)\right|=0 \quad \text { a.s. }
$$

and

$$
\limsup _{n \rightarrow \infty} \sup _{\lambda \in\left[\Lambda^{-1}, \Lambda\right], d>\Delta} y_{n}^{-1} \log \hat{\mu}_{n, d / \lambda}(A \lambda) \leq-\Delta \inf I(A) \quad \text { a.s. }
$$


Proof With $\{$ prop $\}$ denoting the subset of $\Omega$ satisfying the proposition prop, consider for positive $a$ and $b$

$$
C_{a, b, n}^{(i)}:=\left\{\sup _{l \geq a}\left\|Q^{-1} \hat{Q}_{n}\left(\hat{Y}_{n}^{(i)} l^{-1}\right)-Y^{(i)} l^{-1}\right\|_{\infty}>y_{n} b\right\}, \quad i=1, \ldots, n,
$$

which are elements of $\mathcal{F}_{n}$. Furthermore, following (5.8), we can define the empirical probability $\hat{p}_{n}\left(C_{a, b, n}\right):=n^{-1} \Sigma_{i \in\{1, . ., n\}} \mathbf{1}\left(C_{a, b, n}^{(i)}\right)$. Combining Lemmas 2 and 3, we obtain

$$
\lim _{n \rightarrow \infty} y_{n}^{-1} \log \hat{p}_{n}\left(C_{a, b, n}\right)=-\infty \quad \text { a.s. } \quad \text { for all } a, b>0 .
$$

For every $S \subset \mathbb{R}^{m}$ and $\iota>0$, let $S^{\iota}:=\left\{x \in \mathbb{R}^{m}: \inf _{x^{\prime} \in S}\left\|x-x^{\prime}\right\|_{\infty} \leq \iota\right\}$ (closed) and $S^{-\iota}:=\left\{x \in \mathbb{R}^{m}: \inf _{x^{\prime} \in S^{c}}\left\|x-x^{\prime}\right\|_{\infty}>\iota\right\}$ (open). Set $S^{0}:=S$. Since $I$ is a good rate function, Lemma 4.1.6 of Dembo and Zeitouni (1998) implies

$$
\liminf _{\iota \downarrow 0} I\left(A^{\iota}\right)=\inf I(\bar{A})=\inf I\left(A^{o}\right)=\inf I\left(\cup_{\iota>0} A^{-\iota}\right)=\liminf _{\iota \downarrow 0} I\left(A^{-\iota}\right),
$$

so the non increasing function $\iota \mapsto \inf I\left(A^{\iota}\right)$ is continuous in $\left(-\iota_{0}, \iota_{0}\right)$ for some $\iota_{0}>0$, and therefore, $A^{\iota}$ is a continuity set of $I$ for every $\iota \in\left(-\iota_{0}, \iota_{0}\right)$. Moreover, by Eq. 8.36, there exist $\varepsilon>0$ and $\iota_{1} \in\left(0, \iota_{0}\right)$ such that for all $\iota \in\left[0, \iota_{1}\right]$, inf $I\left(A^{-\iota}\right) \leq$ $\inf I(A)+\Delta^{-1} \varepsilon<\Delta^{-1}\left(1-c^{\prime}\right)^{-1}$. Therefore, for

$$
E_{d, \imath, n}^{(i)}:=\left\{Y^{(i)} \in d y_{n} A^{\iota}\right\} \in \mathcal{F}_{n}, \quad i=1, . ., n,
$$

Lemma 1 implies for $\Delta$ satisfying (8.36) and every $\delta \in(0, \Delta)$ and $\iota \in\left[-\iota_{1}, \iota_{1}\right]$ that

$$
\lim _{n \rightarrow \infty} \sup _{d \in[\delta, \Delta]}\left|y_{n}^{-1} \log \hat{p}_{n}\left(E_{d, \iota, n}\right)+d \inf I\left(A^{\iota}\right)\right|=0 \quad \text { a.s., }
$$

and therefore, as $\Delta \inf I\left(A^{-\iota_{1}}\right)<\left(1-c^{\prime}\right)^{-1}$,

$$
\inf _{\iota \in\left[0, \iota_{1}\right]} \liminf _{n \rightarrow \infty} \inf _{d \in[\delta, \Delta]} y_{n}^{-1} \log \hat{p}_{n}\left(E_{d,-\iota, n}\right) \geq-\left(1-c^{\prime}\right)^{-1} \quad \text { a.s. }
$$

Let

$$
D_{\lambda, d, n}^{(i)}:=\left\{\hat{Q}_{n}\left(\hat{Y}_{n}^{(i)} \lambda / d\right) \in Q\left(y_{n} A \lambda\right)\right\} \in \mathcal{F}_{n}, \quad i=1, \ldots, n .
$$

By Eqs. 8.42, 8.39 and 8.45, we have for all $d \geq \delta, \iota>0$ and $\lambda \in\left[\Lambda^{-1}, \Lambda\right]$ that $E_{d,-l, n}^{(i)} \cap\left(C_{d / \lambda, \iota \lambda, n}^{(i)}\right)^{c} \subset D_{\lambda, d, n}^{(i)}$, so $\hat{p}_{n}\left(D_{\lambda, d, n}\right) \geq \hat{p}_{n}\left(E_{d,-\iota, n}\right)-\hat{p}_{n}\left(C_{\delta / \Lambda, \iota / \Lambda, n}\right)$. Therefore, for all $\iota \in\left(0, \iota_{1}\right]$,

$$
\begin{array}{r}
\liminf _{n \rightarrow \infty} \inf _{d \in[\delta, \Delta], \lambda \in\left[\Lambda^{-1}, \Lambda\right]} y_{n}^{-1} \log \hat{p}_{n}\left(D_{\lambda, d, n}\right)-y_{n}^{-1} \log \hat{p}_{n}\left(E_{d,-\iota, n}\right) \\
\geq \liminf _{n \rightarrow \infty} y_{n}^{-1} \log \left(1-\mathrm{e}^{\log \hat{p}_{n}\left(C_{\delta / \Lambda, \iota / \Lambda, n}\right)-\inf _{d \in[\delta, \Delta]} \log \hat{p}_{n}\left(E_{d,-l, n}\right)}\right) \geq 0 \quad \text { a.s., }
\end{array}
$$

the last inequality following from Eqs. 8.40 and 8.44. Therefore, by Eqs. 8.43 and 8.41 ,

$$
\liminf _{n \rightarrow \infty} \inf _{d \in[\delta, \Delta], \lambda \in\left[\Lambda^{-1}, \Lambda\right]} y_{n}^{-1} \log \hat{p}_{n}\left(D_{\lambda, d, n}\right)+d \inf I(A) \geq 0 \quad \text { a.s. }
$$

Furthermore, for all $d \geq \delta, \iota>0$ and $\lambda \in\left[\Lambda^{-1}, \Lambda\right]$, we have $D_{\lambda, d, n}^{(i)} \cap$ $\left(C_{\delta / \Lambda, l / \Lambda, n}^{(i)}\right)^{c} \subset E_{d, l, n}^{(i)}$, so $D_{\lambda, d, n}^{(i)} \subset E_{d, l, n}^{(i)} \cup C_{\delta / \Lambda, \iota / \Lambda, n}^{(i)}$ and therefore,

$$
\hat{p}_{n}\left(D_{\lambda, d, n}\right) \leq 2 \max \left(\hat{p}_{n}\left(C_{\delta / \Lambda, l / \Lambda, n}\right), \hat{p}_{n}\left(E_{d, \iota, n}\right)\right) .
$$


Therefore, by Eqs. 8.40, 8.43 and 8.41,

$$
\limsup _{n \rightarrow \infty} \sup _{d \in[\delta, \Delta], \lambda \in\left[\Lambda^{-1}, \Lambda\right]} y_{n}^{-1} \log \hat{p}_{n}\left(D_{\lambda, d, n}\right)+d \inf I(A) \leq 0 \quad \text { a.s., }
$$

so with Eqs. 8.47 and 8.4, Eq. 8.37 is obtained. By Lemma 1, with $\iota_{0}$ as above,

$$
\limsup _{n \rightarrow \infty} \sup _{d>\Delta} y_{n}^{-1} \log \hat{p}_{n}\left(E_{d, \iota, n}\right) \leq-\Delta \inf I\left(A^{\iota}\right) \quad \text { a.s. }
$$

for all $\iota \in\left[0, \iota_{0}\right)$. Now Eq. 8.38 follows from Eq. 8.48 using Eqs. 8.40, 8.50 and 8.41 .

Acknowledgments The author wants to thank the Associate Editor, two Referees, John Einmahl and Laurens de Haan for their valuable comments and advice, which made the manuscript much better. The support of Rijkswaterstaat by making the oceanographic data available is gratefully acknowledged.

Open Access This article is distributed under the terms of the Creative Commons Attribution 4.0 International License (http://creativecommons.org/licenses/by/4.0/), which permits unrestricted use, distribution, and reproduction in any medium, provided you give appropriate credit to the original author(s) and the source, provide a link to the Creative Commons license, and indicate if changes were made.

\section{Compliance with Ethical Standards}

Conflict of interests The author declares that he has no conflict of interest.

\section{References}

Bingham, N.H., Goldie, C.M., Teugels, J.L.: Regular variation. Cam. Univ. Press (1987)

Broniatowski, M.: On the estimation of the Weibull tail coefficient. J. Stat. Plan. Inference 35, 349-366 (1993)

Bruun, J.T., Tawn, J.A.: Comparison of approaches for estimating the probability of coastal flooding. J. Roy. Statist. Soc. Ser. C 47, 405-423 (1998)

Coles, S.G., Tawn, J.A.: Modelling extreme multivariate events. J. Roy. Statist. Soc. Ser. B 53, 377-392 (1991)

Coles, S.G., Tawn, J.A.: Statistical methods for multivariate extremes: an application to structural design. J. Roy. Statist. Soc. Ser. C 43(1), 1-48 (1994)

Dembo, A., Zeitouni, O.: Large deviations techniques and applications. Springer, New York (1998)

Draisma, G., Drees, H., Ferreira, A., de Haan, L.: Bivariate tail estimation: dependence in asymptotic independence. Bernoulli 10, 251-280 (2004)

Drees, H., de Haan, L.: Estimating failure probabilities. Bernoulli 21(2), 957-1001 (2015)

Einmahl, J.H.J., Lin, T.: Asymptotic normality of extreme value estimators on $C[0,1]$. Ann. Statist. 34, 469-492 (2006)

Embrechts, P., Puccetti, G.: Aggregating risk across matrix structured loss data: the case of operational risk. J. Oper. Risk 3(2), 29-44 (2007)

Ferreira, A., de Haan, L.: The generalised Pareto process; with a view towards application and simulation. Bernoulli 20(4), 171701737 (2014)

de Haan, L., Sinha, A.K.: Estimating the probability of a rare event. Ann. Stat. 27(2), $732-759$ (1999)

de Haan, L., Lin, T.: On convergence toward an extreme value distribution in $C[0,1]$. Ann. Probab. 29(1), 467-483 (2001)

de Haan, L., Ferreira, A.: Extreme value theory - An introduction. Springer (2006)

Hall, P.: On some simple estimates of an exponent of regular variation. J. Roy. Statist. Soc. Ser. B 44(1), 37-42 (1982) 
Heffernan, J.E., Tawn, J.A.: A conditional approach for multivariate extreme values. J.R. Stat. Soc. Ser. B 66, 497-546 (2004)

Heffernan, J.E., Resnick, S.I.: Hidden regular variation and the rank transform. Adv. Appl. Prob. 37(2), 393-414 (2005)

Heffernan, J.E., Resnick, S.I.: Limit laws for random vectors with an extreme component. Ann. Appl. Probab. 17(2), 537-571 (2007)

ISO: Petroleum and natural gas industries - Specific requirements for offshore structures Part 1. ISO/FDIS, 19901-1:2005(E) (2005)

Joe, H., Smith, R.L., Weissman, I.: Bivariate threshold methods for extremes. J. Roy. Statist. Soc. Ser. B 54, 171-183 (1992)

Keef, C., Papastathopoulos, I., Tawn, J.S.: Estimation of the conditional distribution of a multivariate variable given that one of its components is large: additional constraints for the Heffernan and Tawn model. J. Multivariate Anal. 115, 396-404 (2013)

Klüppelberg, C.: On the asymptotic normality of parameter estimates for heavy Weibull-like tails. Preprint (1991)

Ledford, A.W., Tawn, J.A.: Statistics for near independence in multivariate extreme values. Biometrika 83(1), 169-187 (1996)

Ledford, A.W., Tawn, J.A.: Modelling dependence within joint tail regions. J. Roy. Statist. Soc. Ser. B 59, 475-499 (1997)

Ledford, A.W., Tawn, J.A.: Concomitant tail behaviour for extremes. Adv. Appl. Prob. 30, 179-215 (1998)

Peng, L.: Estimation of the coefficient of tail dependence in bivariate extremes. Stat. Probab. Lett. 43, 399-409 (1999)

Pickands, J.: Statistical inference using extreme order statistics. Ann. Stat. 3, 119-131 (1975)

Pickands, J.: Multivariate extreme value distributions. Bulletin of the International Statistical Institute: Proceedings of the 43rd Session (Buenos Aires), 859-878 (1981)

Resnick, S.I.: Extreme values, regular variation, and point processes. Springer (1987)

Resnick, S.I.: Hidden regular variation, second order regular variation and asymptotic independence. Extremes 5, 303-336 (2002)

Ruessink, B.G., Walstra, D.J.R., Southgate, H.N.: Calibration and verification of a parametric wave model on barred beaches. Coast. Eng. 48, 139-149 (2003)

Shorack, G.R., Wellner, J.A.: Linear bounds on the empirical distribution function. Ann. Probab. 6(2), 349-353 (1978)

Smith, R.L., Tawn, J.A., Yuen, H.K.: Statistics of multivariate extremes. Internat. Statist. Rev. 58, 47-58 (1990)

Steenbergen, H.M.G.M., Lassing, B.L., Vrouwenvelder, A.C.W.M., Waarts, P.H.: Reliability analysis of flood defence systems. Heron 49(1), 51-73 (2004)

TAW: Technisch Rapport Golfoploop en Golfoverslag bij Dijken. Technische Adviescommissie voor de Waterkeringen (in Dutch), Delft (2002)

de Valk, C.F.: Approximation of high quantiles from intermediate quantiles (2016). arXiv:1307.6501

Wadsworth, J.L., Tawn, J.A.: A new representation for multivariate tail probabilities. Bernoulli 19(5B), 2689-2714 (2013)

Wellner, J.A.: Limit theorems for the ratio of the empirical distribution function to the true distribution function. Z. Wahrscheinlichkeitstheorie verw. Gebiete 45, 73-88 (1978) 